\title{
Intricate genetic variation networks control the adventitious root growth angle in apple
}

\author{
Caixia Zheng, Fei Shen, Yi Wang, Ting Wu, Xuefeng Xu, Xinzhong Zhang ${ }^{*}$ and Zhenhai Han ${ }^{*}$
}

\begin{abstract}
Background: The root growth angle (RGA) typically determines plant rooting depth, which is significant for plant anchorage and abiotic stress tolerance. Several quantitative trait loci (QTLS) for RGA have been identified in crops. However, the underlying mechanisms of the RGA remain poorly understood, especially in apple rootstocks. The objective of this study was to identify QTLS, validate genetic variation networks, and develop molecular markers for the RGA in apple rootstock.

Results: Bulked segregant analysis by sequencing (BSA-seq) identified 25 QTLs for RGA using 1955 hybrids of the apple rootstock cultivars 'Baleng Crab' (Malus robusta Rehd., large RGA) and 'M9' (M. pumila Mill., small RGA). With RNA sequencing (RNA-seq) and parental resequencing, six major functional genes were identified and constituted two genetic variation networks for the RGA. Two single nucleotide polymorphisms (SNPS) of the MdLAZY1 promoter damaged the binding sites of MdDREB2A and MdHSFB3, while one SNP of MdDREB2A and MdIAA1 affected the interactions of MdDREB2AMMdHSFB3 and MdIAA1/MdLAZY1, respectively. A SNP within the MdNPR5 promoter damaged the interaction between MdNPR5 and MdLBD41, while one SNP of MdLBD41 interrupted the MdLBD41/MdbHLH48 interaction that affected the binding ability of MdLBD41 on the MdNPR5 promoter. Twenty six SNP markers were designed on candidate genes in each QTL interval, and the marker effects varied from $0.22^{\circ}-26.11^{\circ}$.
\end{abstract}

Conclusions: Six diagnostic markers, SNP592, G122, b13, Z312, S1272, and S1288, were used to identify two intricate genetic variation networks that control the RGA and may provide new insights into the accuracy of the molecular markers. The QTLs and SNP markers can potentially be used to select deep-rooted apple rootstocks.

Keywords: Malus, RGA, BSA-seq, RNA-seq, QTLs, Genetic variation

\section{Background}

Apple (Malus domestica Borkh.) is one of the predominant fruit crops. Dwarfing rootstock is widely used as an ideal method for achieving high-density planting of apple [1]. However, apple trees that are grafted on dwarfing rootstocks usually have a relatively shallow root system, which leads to poor anchorage and increased sensitivity to ambient stress from, for example, wind or water logging [2]. Moreover, plants with shallow roots are often vulnerable to environmental stresses such as drought, cold, and salinity [3, 4],

*Correspondence: zhangxinzhong999@126.com; rschan@cau.edu.cn College of Horticulture, China Agricultural University, Beijing 100193, China whereas deep roots are pivotal for water uptake, nutrient uptake, and adaptability [5]. The depth of the root architecture is determined by the root growth angle (RGA), which is defined as the angle between the direction of root growth and the horizontal axis $[6,7]$. However, the understanding of the development and underlying mechanisms of the RGA in apple lag far behind that in cereal crops [8-10].

Gravity, light, and water potential are major environmental factors that affect the RGA [11]. The perception and response of plant roots to gravity have been described as gravitropic $[12,13]$. Two different mechanisms of gravity signal transduction are active in root columella cells and in the root elongation zone [14]. The starch-statolith

(c) The Author(s). 2020 Open Access This article is licensed under a Creative Commons Attribution 4.0 International License, which permits use, sharing, adaptation, distribution and reproduction in any medium or format, as long as you give appropriate credit to the original author(s) and the source, provide a link to the Creative Commons licence, and indicate if changes were made. The images or other third party material in this article are included in the article's Creative Commons licence, unless indicated otherwise in a credit line to the material. If material is not included in the article's Creative Commons licence and your intended use is not permitted by statutory regulation or exceeds the permitted use, you will need to obtain permission directly from the copyright holder. To view a copy of this licence, visit http://creativecommons.org/licenses/by/4.0/. The Creative Commons Public Domain Dedication waiver (http://creativecommons.org/publicdomain/zero/1.0/) applies to the data made available in this article, unless otherwise stated in a credit line to the data. 
hypothesis indicates that the columella cells of plant roots sense the direction of gravity by sedimentation of amyloplasts and can thus maintain positive gravitropism. Consequently, amyloplasts are always at the cell bottom matching the direction of gravity $[15,16]$. A different gravitropic sensing hypothesis states that the cells of the root elongation zone also sense gravity and bend following the direction of gravity without the use of amyloplasts [14]. The RGA is also easily affected by light, which suggests that phototropism governs the RGA [17]. The RGA in phytochrome A (phyA) mutants of Arabidopsis responds positively to red light and is negatively phototropic to blue light in phototropin1 (phot1) mutants [18-20]. However, water potential is another factor that affects the RGA, and this response of plant roots is often called hydrotropism [21, 22]. Several mutants in Arabidopsis, such as none hydrotropic response1 (nhr1) or mizu-kussei1 (miz1), and altered hydrotropic response1 (ahr1), demonstrated varied hydrotropic responses to water gradients [21, 23-25]. LONG HYPOCOTYL 5 (HY5)-mediated light signalling has been shown to enhance the hydrotropism of MIZ1, which initiates downward growth of plant roots and leads to the development of a larger root system in Arabidopsis [26].

The mechanism of root gravitropism has demonstrated that on the opposite sides of plant roots, different levels of auxin accumulation lead to an asymmetric elongation zone of cells, which causes gravitropic bending of the roots [13, $27,28]$. In response to this gravitropic bending of roots, the auxin-influx carrier AUXIN RESISTANT 1 (AUX1) and auxin-efflux carriers PIN2, PIN3, and PIN7 are the central players [29-33]. Arabidopsis mutants with defective gravitropic responses are generated via genetic mutations in the auxin signalling pathway, mediated by interactions of both AUXIN (AUX)/INDOLE-3-ACETIC ACID (IAA) and AUXIN RESPONSE FACTOR (ARF) [34-37].

Among the mutations causing defective gravitropic responses in many plant species, lazy1 mutations often lead to an asymmetric auxin distribution in either roots or shoots [38-42]. The functions of LAZY1 can be affected via interaction with IAA1, HEAT STRESS TRANSCRIPTION FACTOR 2D (HSFA2D), and Brevis Radix 4 (BRXL4) by altering the responses to auxin signalling [43-45]. OsIAA mutants in rice (Oryza sativa L.) have a single amino acid substitution in the core sequence of domain II, which blocks auxin signalling, and the resulting mutant plants displayed phenotypes defective in gravitropic responses [46-48]. Overexpression of the dehydration-responsive element-binding protein $2 \mathrm{~A}$ (DREB2A) gene, which specifically binds to the sequence 5 '[A/G]CCGAC-3', led to more vertical roots in transformants than in the wild type (WT) $[49,50]$. Lateral organ boundary domain-containing proteins (LBD) are dominant regulators of the formation of adventitious roots and the development of other lateral organs [51-53]. In Arabidopsis, LBD adaxialabaxial polarity can be affected by 35S:BOP1 transgenesis, and the resulting transformants exhibited a downwardorienting silique phenotype [54, 55]. The interaction of basic helix-loop-helix 48 (bHLH48) with the C-terminus of LBD may affect the capability of LBD to bind to the target gene promoter sequence $5^{\prime}-(\mathrm{G}) \mathrm{CGGC}(\mathrm{G})-3^{\prime}$ and may thus regulate the development of lateral organ boundaries [56-59].

To identify natural genetic variations that are involved in plant RGA, many quantitative trait loci (QTLs) have been reported in a diversity of Poaceae species, such as Zea hybrids [9], sorghum (Sorghum bicolor L.) [10, 60], rice [7, 8, 61-64], and wheat (Triticum aestivum L.) [65, 66]. Recently, in rice, several candidate genes, such as $D R O 1, D R O 2$, and qSOR1, were predicted within QTL regions on chromosomes 9, 4, and 7, respectively [61-63]. However, QTLs for RGA have not been identified in woody perennials, and the molecular genetic control of RGA remains to be elucidated.

Marker-assisted selection (MAS) aims to predict the performance of a trait of an offspring by one or few markers with major effects [67]. Root morphology and drought resistance in Kalinga III rice were greatly enhanced by MAS in a backcross population [68]. Four root trait QTLs were introduced into upland rice by using MAS to increase yield [69]. In a scenario where a trait is associated with up to thousands of genes with minor or infinitesimal effects, MAS will be ineffective. Thus, the genomic selection (GS) strategy emerged, which considers the impact of all available genetic markers for the prediction of breeding value [70]. The advantage of GS is to estimate the effects of all the markers on the target trait, e.g., genomic breeding value estimation [71, 72]. Conventionally detected significant QTLs may have distinct large effects in GS models. Significant root trait-associated QTLs have been detected in Brassica napus [73, 74]. Five significant root length QTLs were identified across four chromosomes in wheat by using genome-wide association mapping (GWAS) with a total of 25,125 marker pairs [75]. In contrast, the large effect markers related to root architecture were also selected in wheat by using a mixed linear model of GWAS with single nucleotide polymorphism (SNP) arrays [76].

The objective of this study was to identify QTLs and subsequent candidate genes that are associated with apple rootstock RGA via BSA-seq. Then, the interactions between candidate genes were verified to obtain diagnostic markers and to decode the genetic variation network of the RGA. Furthermore, the effects of QTL-based markers on RGA were estimated.

\section{Results}

Inheritance of apple adventitious RGA and construction of extreme RGA phenotype bulks

Phenotype data, in the form of the average RGA of three leafy cuttings of 1955 and 1383 hybrids derived from 
'Baleng Crab (BC)' (Malus robusta Rehd.) × 'M9' (M. pumila Mill.), were obtained in 2016 and 2017, respectively (Table S1). The RGA of the hybrids segregated significantly by $9.26-58.95^{\circ}$ (Table S1). The frequency distribution was not a typical Gaussian distribution, and the broad-sense heritability was $85.26 \%$ in both years (Fig. S1A and B).

Using the total mean RGA of the two experimental years, genomic DNA of 30 hybrids with extremely large mean RGAs $\left(53.43^{\circ}\right)$ and 30 hybrids with extremely small mean RGAs $\left(12.70^{\circ}\right)$ were mixed to construct two extreme bulks. The mean RGA values of the two extreme bulks were significantly different from each other (Fig. S1C and D).

\section{Identification of QTLs for RGA}

A total of 237,892,668 clean reads were identified in both extreme RGA bulks (Table S2). In small and large RGA bulks, 97.38 and $96.86 \%$ of the clean reads were mapped to the Malus $\times$ domestica genome GDDH13 v1.1 (GDDH13, https://iris.angers.inra.fr/gddh13/), respectively. Only uniquely mapped reads were used for further analysis at mapping rates of 75.83 and $78.46 \%$ for small and large RGA bulks, respectively (Table S2).

Twenty-five significant QTLs were identified according to the criterion of a false discovery rate $(F D R)<0.01$ (Table S3). Eleven of these QTLs were mapped on the maternal parent ' $\mathrm{BC}$ ' (headed by $\mathrm{B}$ ), eight were mapped on the pollen parent 'M9' (headed by M), and six were located on both parents (headed by $\mathrm{H}$ ), with significant thresholds of 2.93 , 3.13, and 3.38, respectively (Fig. S2; Table S3).

By changing the sliding window sizes of $1.25,1.00$, $0.75,0.50$, and $0.25 \mathrm{Mb}$ (mega base pair), the stabilities of the QTL regions were verified, and the QTL intervals were narrowed from 31.34 to $28.14 \mathrm{Mb}$. The average G' scores increased from 3.55 to 7.01 (Table S3).

\section{Analysis of transcriptome data}

RNA sequencing (RNA-seq) yielded 19,935,274 clean reads from the 24 samples derived from cuttings of each of the three 'BC' $\times$ 'M9' hybrids with extremely small or extremely large RGAs. Samples were assessed at 0, 7, 14, and $21 \mathrm{~d}$ after cutting. Of these reads, more than $82.73 \%$ were uniquely mapped to the apple genome GDDH13 (Table S4, S5). In the RNA-seq data of extremely small and large RGAs, the correlation coefficients of three analogous hybrids were 0.72-0.97 at the same sampling time points (Table S6).

A total of 2849 differentially expressed unigenes (DEGs) with 2-fold changes $(\log 2>1$ or $\log 2<-1$ and FDRs $<$ 0.05 between samples were found based on their fragments per kilobase per million mapped reads (FPKM) values in both extreme bulks during the four sampling time points. A total of 1239 (43.49\%), 296 (10.39\%), 330 (11.58\%), and 1257 (44.12\%) DEGs were distributed in samples from $0,7,14$, and $21 \mathrm{~d}$, respectively (Fig. S3A).
In the gene ontology (GO) classification, 1225 DEGs $(43.00 \%)$ were identified, and more than half of these (54.68\%) were enriched in the molecular function category (Fig. S4). Moreover, 706 (78.88\%) DEGs were classified into metabolic pathways according to the Kyoto Encyclopedia of Genes and Genomes (KEGG) classification (Fig. S5A and B). In the KEGG enrichment analysis, 11 pathways were selected via $P$-values $<0.01$, including four pathways that were closely associated with RGA, plant hormone signal transduction (60 DEGs), starch and sucrose metabolism (52 DEGs), terpenoid backbone biosynthesis (20 DEGs), and alpha-linolenic acid metabolism (21 DEGs) (Fig. S5C and D).

Of the 60 DEGs in the plant hormone signalling pathway, 30 were involved in auxin signalling (Table S7). Twentyone auxin signalling genes (including SAUR, AUX/IAA, $G H 3$, and TIR1) and two gibberellin signalling-related phytochrome interacting factor genes (MD09G1146000 and MD17G1132600) had significantly higher expression levels $(\log 2>1$ and FDR $<0.05)$ in samples with large RGAs than in samples with small RGAs at $0 \mathrm{~d}$ (Fig. S3B; Table S7). Furthermore, two auxin signalling-related $A R F$ and $L A X$ genes (MD15G1014400 and MD12G1162400), two cytokinin signalling-related ARR-B family genes (MD1 3G1108300 and MD16G1159400), and four abscisic acidrelated protein phosphatase 2C genes (MD01G1220800, MD03G1085400, MD07G1291000, and MD11G1093100) were significantly less expressed $(\log 2<-1$ and FDR $<$ 0.05 ) in samples with large RGAs than in those with small RGAs at $0 \mathrm{~d}$ (Fig. S3B; Table S7). However, three auxin signalling genes related to AUX/IAA and GH3 genes (MD09G1208000, MD17G1189100 and MD05G1092300), two cytokinin signalling-related histidine-containing phosphotransferase protein genes (MD03G1272900 and MD11G1293900), and three abscisic acid receptor PYR/ PYL family genes (MD01G1158500, MD07G1227100, and MD12G1178800) had significantly higher expression in cuttings with large RGAs at $21 \mathrm{~d}$ (Fig. S3B; Table S7).

Of the 52 DEGs in the starch and sucrose metabolism pathways, 13 were significantly highly expressed at $0 \mathrm{~d}$, whereas 15 were significantly less expressed in large RGA cuttings at $0 \mathrm{~d}$. However, eight and five genes had significantly lower and higher expression levels in large RGA cuttings at $21 \mathrm{~d}$, respectively (Fig. S3C; Table S7). Eighteen of the 20 DEGs related to terpenoid backbone biosynthesis were expressed at significantly higher levels in large RGA cuttings at $21 \mathrm{~d}$ (Fig. S3D; Table S7). Of the 21 DEGs involved in alpha-linolenic acid metabolism, nine and seven were significantly highly expressed in large RGA cuttings at $0 \mathrm{~d}$ and $21 \mathrm{~d}$, respectively (Fig. S3E; Table S7).

To link the DEGs and the candidate genes from the QTL intervals, a co-expression network was analysed using AppleMDO tools (http://bioinformatics.cau.edu. cn/AppleMDO/?from=groupmessage) (Table S8). The 
SAUR-like auxin-responsive gene MD10G1060900, within the interval of QTL M10.1, was positively coexpressed with seven auxin-responsive DEGs at $0 \mathrm{~d}$. Two genes from QTL B03.1, MD03G1292900, and MD03G1288700, which encode a WRKY family transcription factor and a photolyase/blue-light receptor 2, were positively co-expressed with two gibberellinresponsive and two terpenoid backbone biosynthesisrelated DEGs at $0 \mathrm{~d}$. A CBL-interacting protein kinase 21 gene, MD13G1265500, which is located at the QTL region of H13.1, may positively regulate four DEGs in starch and sucrose metabolism.

\section{Prediction of candidate genes}

From the narrowed regions of the 25 identified QTLs, 2645 genes with 87,576 SNPs and 250 structural variations (SVs) were selected from parental resequencing data (Table S9). Of these genes, 576 were excluded because their SNPs or SVs did not affect the cis-element on the upstream sequence or the functional domain on the coding region (Table S9). Another 536 genes were not expressed throughout the stem tissue, and 421 genes, with SNPs or SVs only within the promoter, did not show differential expression between cuttings with large and small RGAs (Table S9). Additionally, 828 genes were excluded from further analysis since their functional annotation or subcellular localization were not closely related to the RGA, as identified via the UniProt database (http://www.uniprot.org/) (Table S9). Finally, 284 genes were selected as candidates that are likely involved in RGA (Table S10). Based on the functional annotation and the published data from the other authors [41, $43,44,49,51,55]$, the following six genes are potentially involved in the regulation of the RGA and thus were selected for further functional analysis: MdNPR5 (MD09G1083600), MdLBD41 (MD09G1088700), MdLAZY1 (MD13G112240 0), MdbHLH48 (MD14G1064200), MdDREB2A (MD17G1 089700), and MdIAA1 (MD17G1198300).

\section{Experimental validation of candidate genes Allelic variation of MdLAZY1 positively affects the RGA} MdLAZY1 was predicted near the peak of QTL B13.2 (Table S10). The expression levels of MdLAZY1 in the stem tissue of hybrids with large RGAs were significantly higher than those in hybrids with small RGAs at $0 \mathrm{~d}$ after cutting (Fig. 1a). Apart from three synonymous SNPs at the coding sequence (CDS), there were also two completely linked G/A SNPs at $-1485 \mathrm{bp}$ and $-474 \mathrm{bp}$ upstream of the ATG codon (SNP-1485/- 474 G/A) (Fig. 1b; Table S11; Supplementary File 1). These two SNPs alter the cis-elements of DRE and HSF within the promoter of MdLAZY1. Kompetitive allele-specific PCR (KASP) assay of 265 F1 hybrids showed that the RGA of hybrids with the $\mathrm{T}: \mathrm{G}$ genotype of marker b13, which was linked with SNP-1485/-474G:A, was significantly higher than those with the T:T genotype of b13, which was linked with the SNP-1485/- 474 G:G (Fig. 1c; Table S12).

Truncating the DRE cis-element or both DRE and HSF cis-elements led to an additive increase in the luciferase (LUC) activities of MdLAZY1-pro-MG:LUC and MdLA ZY1-pro-BG:LUC, rather than MdLAZY1-pro-BA:LUC (Fig. 1d and e). These data indicated that the cis-element of DRE and HSF negatively affected the expression of MdLAZY1. Allele A of SNP-1485/-474 caused functional deficiency in DRE and HSF cis-elements and increased the expression levels of MdLAZY1 and the RGA.

Subcellular localization showed that MdLAZY1 was localized in the nucleus and plasma membrane (Fig. 1f). Moreover, 35S:MdLAZY1 transgenic Nicotiana benthamiana lines were obtained and confirmed by PCR at the DNA and cDNA levels (Fig. S6A). A significant increase in RGA (by a mean of $12.28^{\circ}$ ) was observed in 35S:MdLAZY1 transformants compared with that of the WT (Fig. 1g). When 35S:MdLAZY1 and $p T R V: M d L A Z Y 1$ were transiently transformed into hybrids from 'BC' $\times$ 'M9' with small and large RGAs, the relative expression levels of $M d M$ dIAA1, MdDREB2A, and MdHSFB3 did not show significant changes compared with those of untransformed hybrids (Fig. $1 \mathrm{~h}$ and i). These data indicated that MdLAZY1 functions downstream of $M d M d I A A 1, M d D R E B 2 A$, and MdHSFB3.

\section{Allelic variations of MdIAA1, MdDREB2A, and MdHSFB3}

MdIAA1 (QTL M17.1) showed significant protein sequence alignment with IAA17 of Arabidopsis thaliana (https://www.arabidopsis.org/Blast/index.jsp) $\quad$ (e-value = 4e-63) (Table S10). No significant differences in the relative expression of MdIAA1 were detected $0-21 \mathrm{~d}$ after cutting between stem tissues of F1 hybrids with large and small RGAs (Fig. 2a). The full-length CDS of MdIAA1 comprises four exons, encoding a 205-aa protein. An A/G variant was detected at +223 bp downstream of the ATG codon (SNP223 A/G). SNP223 resulted in an amino acid substitution from lysine to glutamate at the conserved domains. Another SNP274 $\mathrm{A} / \mathrm{T}$ at the second exon (which causes amino acid substitution) was homozygous $\mathrm{A}: \mathrm{A}$ in 'BC' and homozygous T:T in 'M9'. Consequently, SNP274 may not segregate in the F1 population (Fig. 2d; Table S11; Supplementary File $2 \mathrm{~A}$ and $\mathrm{B}$ ). The RGA of 50 hybrids with the A:C genotype of the KASP marker G122, which was linked with SNP223 A:G, was significantly lower than that of 212 hybrids with the A:A genotype of G122, which was linked with SNP223 A:A (Fig. 2g; Table S12).

The MdDREB2A gene was predicted from QTL B17.1 (Table S10). No significant differences were detected in the relative expression of MdDREB2A between cuttings of F1 hybrids with small and large RGAs at any time point after cutting (Fig. 2b). The MdDREB2A gene, which did 


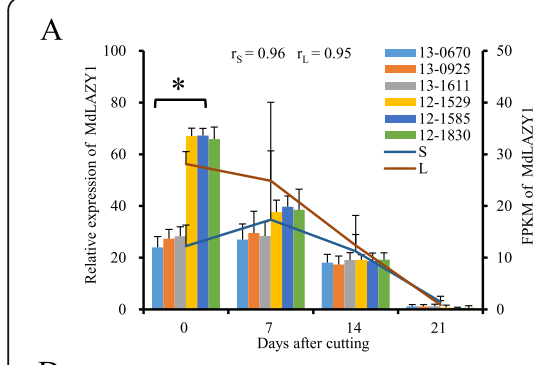

D

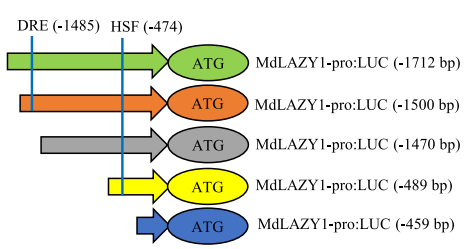

G

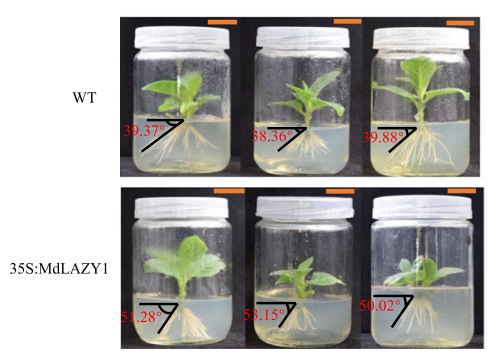

B

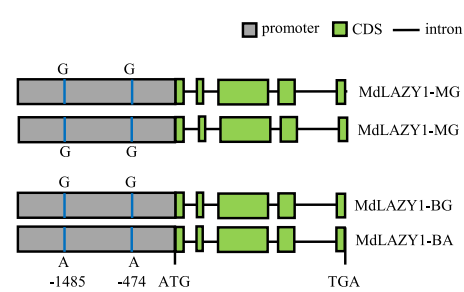

E

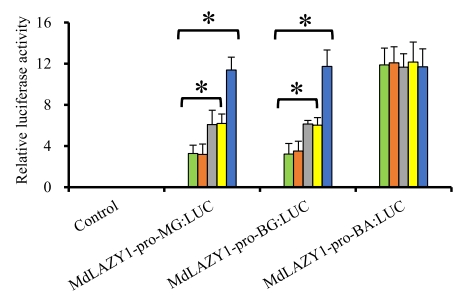

$\mathrm{H}$

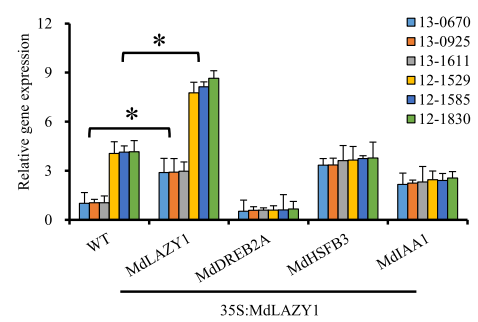

C

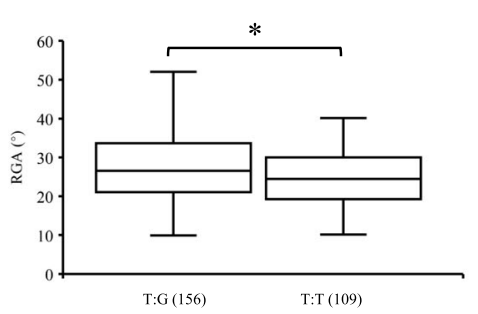

F

F $\quad$ GFP Merged

Bright Field

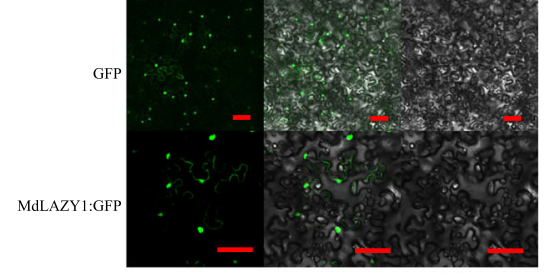

I

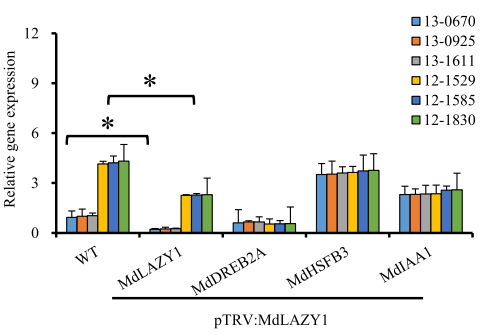

Fig. 1 Verification of the allelic variations in the promoter of MdLAZY1 and functional analysis of 'Baleng Crab (BC)' (Malus robusta), 'M9' (M. pumila) and their F1 hybrids. a MdLAZY1 expression by real-time quantitative PCR (RT-qPCR) (bar chart) and mean fragments per kilobase per million mapped reads (FPKM) values (line chart) of the stem tissue of leafy cuttings during adventitious root formation in three hybrids (13-0670, 13-0925, and 13-1611) with small (S) and three hybrids (12-1529, 12-1585, and 12-1830) with large (L) RGAs. b Structural variations of MdLAZY1. c Box plot showing differences in RGAs between hybrids with the T:G and T:T genotypes of marker b13. Numbers of the hybrids are presented in parentheses. $\mathbf{d}$ Schematic representation of MdLAZY1-pro: LUC vectors truncated with or without DRE and HSF cis-elements. "pro" represents the promoter. e Transient expression of MdLAZY1-pro: LUC variants and truncates, which were truncated as described in panel $\mathbf{d}$. e Subcellular localization of transiently expressed MdLAZY1:GFP in Nicotiana benthamiana. Scale bars $=50 \mathrm{~mm}$. g RGA phenotypes in the 35S:MdLAZY1 transgenic $N$. benthamiana lines and untransformed wild type. $\mathbf{h}$ and (i) Relative expression of MdIAA1, MdDREB2A, and MdHSFB3 when MdLAZY1 was transiently transformed by the vectors 35S:MdLAZY1 (H) and PTRV:MdLAZY1 (i). Asterisks represent $P<0.05$ by Dunnett's multiple comparison

not contain an intron, had the following six nonsynonymous SNPs: SNP25 A/T, SNP27 A/G, SNP425 C/G, SNP515 C/T, SNP551 C/T, and SNP592 T/C. Of these six SNPs, SNP592 caused a stop loss variation with a five amino acid extension (Fig. 2e; Table S11; Supplementary File $2 \mathrm{C}$ and D). Significantly smaller RGA values were detected in 165 hybrids with $\mathrm{T}: \mathrm{C}$ than in 99 hybrids with the $\mathrm{T}: \mathrm{T}$ genotype of SNP592 (Fig. 2h).

MdHSFB3 (MD14G1075400) was not located in the intervals of any QTL. The expression of MdHSFB3 was higher at day 0 but was lower at day 21 after cutting in the stem tissues of hybrids with small RGAs than in those with large RGAs (Fig. 2c). MdHSFB3 encodes a 163-aa protein, and SNP324 C/A of MdHSFB3 resulted in an amino acid substitution from aspartate to glutamate (Fig. 2f; Table S11; Supplementary File 2E and F).

\section{Non-allelic interaction of MdLAZY1, MdIAA1, MdDREB2A, and MdHSFB3}

A yeast two-hybrid ( $\mathrm{Y} 2 \mathrm{H})$ assay and bimolecular fluorescence complementation (BiFC) showed that MdLAZY1 interacted with MdIAA1-MG but not with MdIAA1-MA or MdIAA1-BA (Fig. 3a; Fig. S7A). The joint effect of MdLAZY1 SNP-1485/- 474 G:A and MdIAA1 SNP223 A: $\mathrm{G}$ was $-4.85^{\circ}$, which is far lower than their individual effects (Fig. 3b; Table S13). These data indicated an obvious epistasis of the MdIAA1 SNP223 G allele on allele A of SNP-1485/- 474 in the MdLAZY1 promoter.

$B D$-MdLAZY1-MG or BD-MdLAZY1-BG (but not $B D$ MdLAZY1-BA) interacted with all genotypes of $A D$ MdDREB2A and $A D$-MdHSFB3 as identified by yeastone-hybrid $(\mathrm{Y} 1 \mathrm{H})$ assay (Fig. 3c). Coinjection of 35S: MdDREB2A or 35S:MdHSFB3 with MdLAZY1-pro-MG: $L U C$ and MdLAZY1-pro-BG:LUC, instead of MdLAZY1- 


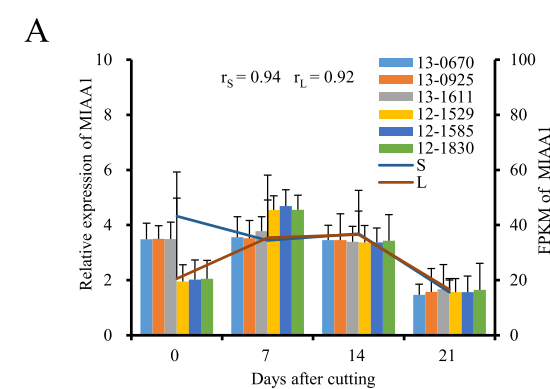

B

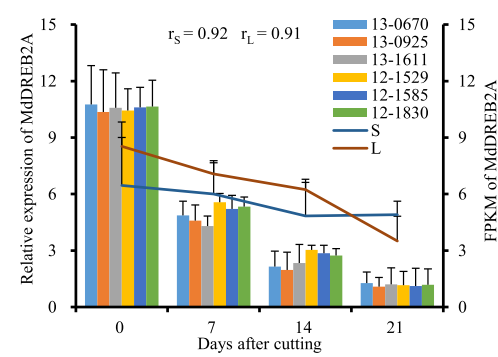

$\mathrm{C}$

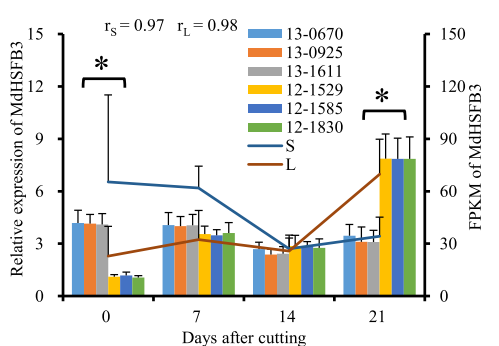

$\mathrm{D}$

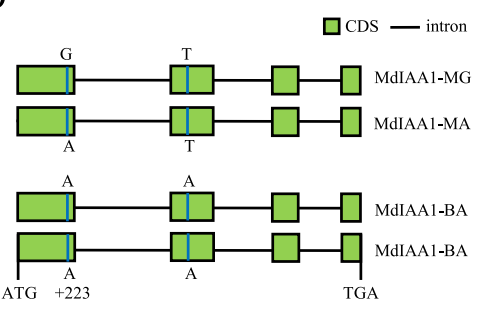

E

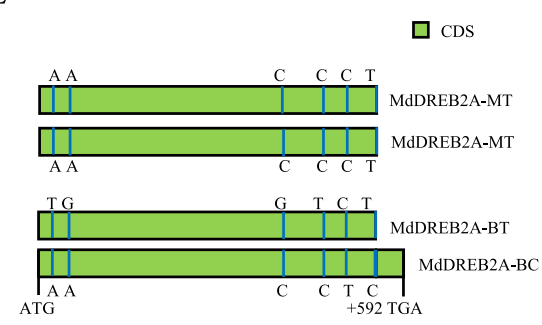

$\mathrm{F}$

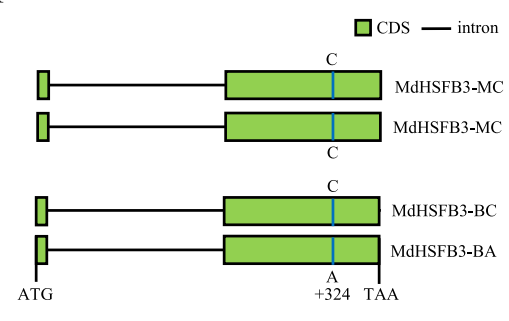

G

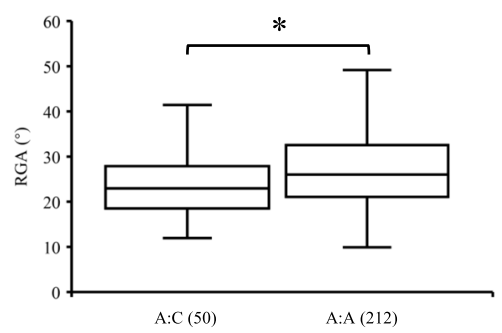

$\mathrm{H}$

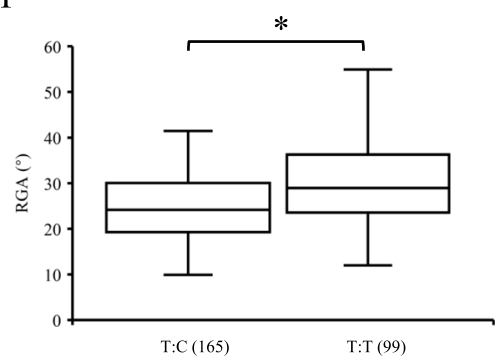

Fig. 2 Allelic variations and the expression patterns of MdIAA1, MdDREB2A, and MdHSFB3 in 'BC', 'M9' and their F1 hybrids. a-c Expression of MdIAA1 (a), MdDREB2A (b), and MdHSFB3 (c) by RT-qPCR (bar chart) and mean FPKM values (line chart) during adventitious root formation in hybrids with small (S) (13-0670, 13-0925, and 13-1611) and large (L) (12-1529, 12-1585, and 12-1830) RGAs, respectively. (D-F) Schematic of single nucleotide variations of MdIAA1 (d), MdDREB2A (e), and MdHSFB3 (f). (g) and (h) Box plots showing RGA differences of markers G122 (g) and SNP592 (h), respectively. Numbers of the hybrids are given in parentheses. Asterisks represent P < 0.05 by Dunnett's multiple comparison

pro-BA:LUC, resulted in lower LUC activity than that of the control (Fig. 3d). These data further validated that MdDREB2A and MdHSFB3 negatively regulated the expression of $M d L A Z Y 1$ via the cis-element of DRE and HSF.

MdDREB2A-BC, rather than MdDREB2A-BT or MdDR EB2A-MT, interacted with MdHSFB3-BA and MdHSFB3$\mathrm{MC}$, as identified via $\mathrm{Y} 2 \mathrm{H}$ and BIFC assays. However, a point mutation of MdDREB2A-BC (592 C to T), MdDR EB2A-BC (p), could not interact with MdHSFB3 at all (Fig. 3e and Fig. S7B). These results indicated that the SNP592 T/C of MdDREB2A altered its ability to interact with MdHSFB3. Coinjection of MdLAZY1-pro-MG:LUC or MdLAZY1-pro-BG:LUC (but not MdLAZY1-pro-BA:LUC) with 35S:MdHSFB3-BA/MC + 35S:MdDREB2A-BC led to significantly lower LUC activity compared with both the control and samples coinjected with 35S:MdHSFB3-BA/ $\mathrm{MC}+35 S: M d D R E B 2 A-B T / M T$ (Fig. 3f). The interaction between MdDREB2A SNP592 allele C and MdHSFB3 enhanced the negative regulation of $M d L A Z Y 1$ promoter activity of SNP-1485/- 474 allele G.

The combination of the SNP223 A:A of MdIAA1 and the SNP592 T:T of MdDREB2A exhibited larger genotype effects $\left(4.82^{\circ}\right.$ and $\left.6.14^{\circ}\right)$ than those with one or two heterozygous genotypes, irrespective of $M d L A Z Y 1$ genotype (Fig. 3g, Table S13). Therefore, MdDREB2A and MdIAA1 exhibited epistatic non-allelic effects on MdLAZY1 (Fig. $3 \mathrm{~h}$ and i).

\section{Allelic variation of MdNPR5 negatively affects $R G A$}

MdNPR5 was in QTL B09.1 (Table S10). The relative expression of MdNPR5 was significantly higher in the stem tissues of cuttings with small RGAs than in those with large RGAs at day 0 after cutting (Fig. 4a). MdNPR5 encoded a 61-aa protein without nonsynonymous variations in the unique exon. However, SNP-1228 G/A, which alters the LBD cis-element, was found within the promoter of MdNPR5 (Fig. 4b; Table S11; Supplementary 


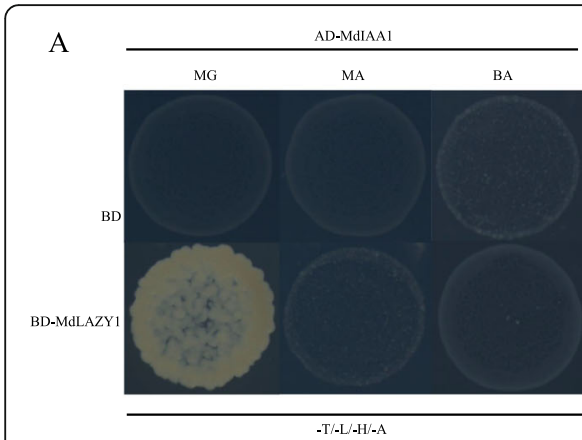

B

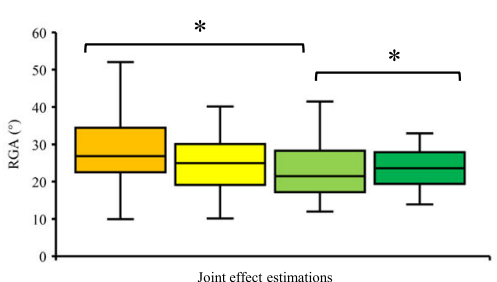

$\mathbf{Q} \mathrm{G} 122(\mathrm{~A}: \mathrm{A})+\mathrm{b} 13(\mathrm{~T}: \mathrm{G})(125) \quad \mathbf{G} 122(\mathrm{~A}: \mathrm{A})+\mathrm{b} 13(\mathrm{~T}: \mathrm{T})(86)$ 口 G122 (A:C) + b13 (T:G) (28) पG122 (A:C) + b13 (T:T) (22)

G

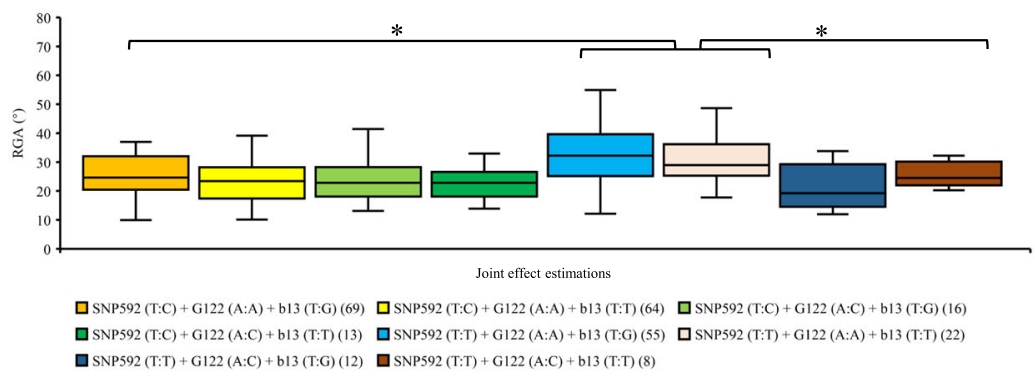

$\mathrm{C}$

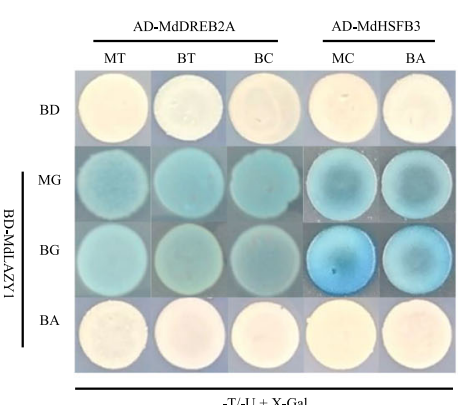

D
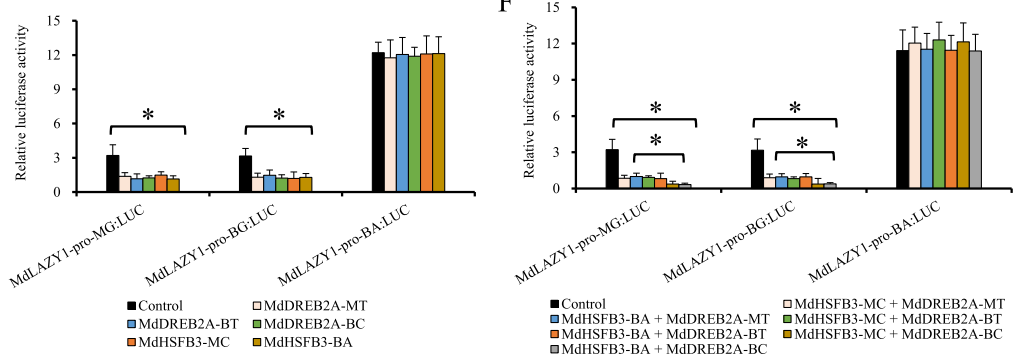

$\mathrm{H}$

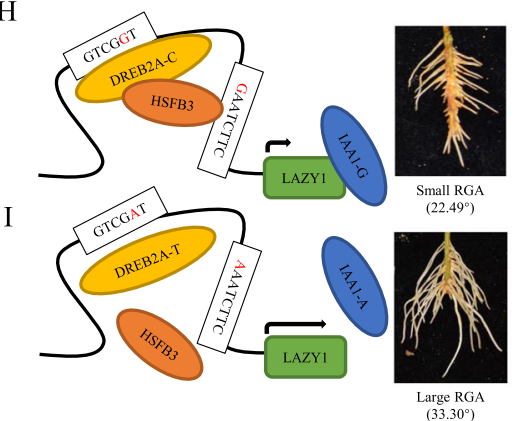

Fig. 3 Non-allelic interactions between MdLAZY1, MdIAA1, MdDREB2A, and MdHSFB3 and their effects on adventitious RGA. a Yeast two-hybrid $(\mathrm{Y} 2 \mathrm{H})$ assay showing protein-protein interactions between MdLAZY1 and MdIAA1. b and (g) Joint genotype effect estimations of markers G122/ b13 (b) and G122/b13/SNP592 (g). Numbers of the hybrids are presented in parentheses. c Yeast-one-hybrid (Y1H) images showing the interactions of the MdLAZY1 promoter with the proteins MdDREB2A and MdHSFB3. $\mathbf{d}$ Transient coexpression analysis of MdLAZY1-pro: LUC interacting with 35S:MdDREB2A or 35S:MdHSFB3. e Y2H assay showing protein-protein interactions between MdDREB2A and MdHSFB3. BDDREB2A-BC ( $p$ ) indicates a point mutation of C (SNP592) to T in MdDREB2A-BC. $\mathbf{f}$ Transient coexpression analysis of MdLAZY1-pro: LUC interacting with 35S:MdDREB2A and 35S:MdHSFB3. Asterisks represent $P<0.05$ by Dunnett's multiple comparison. $\mathbf{h}$ and (i) Proposed model for the genetic variation network regulating RGA. The arrow length indicates the gene expression level. Numbers of the hybrids are presented in parentheses

File 3). The KASP assay showed that the average RGA of 81 F1 hybrids with the SNP-1228 G:A genotype, linked by the C:T of marker S1272, was significantly larger than that of 177 hybrids with the SNP-1228 G:G genotype, linked with the C:C of marker S1272 (Fig. 4c; Table S12).

A promoter truncation assay showed that the LUC activity of both full-length and truncated MdNPR5-proMG and MdNPR5-pro-BG (including - 1228 G) was significantly higher than that of MdNPR5-pro-BA and truncated the $-1228 \mathrm{G}$ allele in MdNPR5-pro-MG and MdNPR5-pro-BG (Fig. 4d and e). These data indicated that the LBD cis-element exerted a positive effect on MdNPR5 transcription and that SNP-1228 allele A completely destroyed the LBD cis-element.

Subcellular localization analysis identified GFP signals of the MdNPR5 fused protein in the nucleus (Fig. 4f). In addition, the 35S:MdNPR5 transgenic $N$. benthamiana lines exhibited a significant decrease in RGA $\left(10.76^{\circ}\right.$ by mean) compared with that of the WT (Fig. 1g; Fig. 4g; Fig. S6B). The 35S:MdNPR5 and $p T R V$ :MdNPR5 transiently transformed hybrids with small and large RGAs from 'BC' $\times$ 'M9' did not show significant differences in the relative expression levels of $M d M d L B D 41$ and MdbHLH48 compared with those of untreated hybrids (Fig. $4 \mathrm{~h}$ and i). These data indicated that MdNPR5 acted downstream of MdMdLBD41 and MdbHLH48.

\section{Allelic variations and effects of MdLBD41 and MdbHLH48 on RGA}

MdLBD41 was located at the peak of QTL B09.1 (Table S10). MdLBD41 had significantly higher relative expression and FPKM values in the stem tissue of hybrids with 


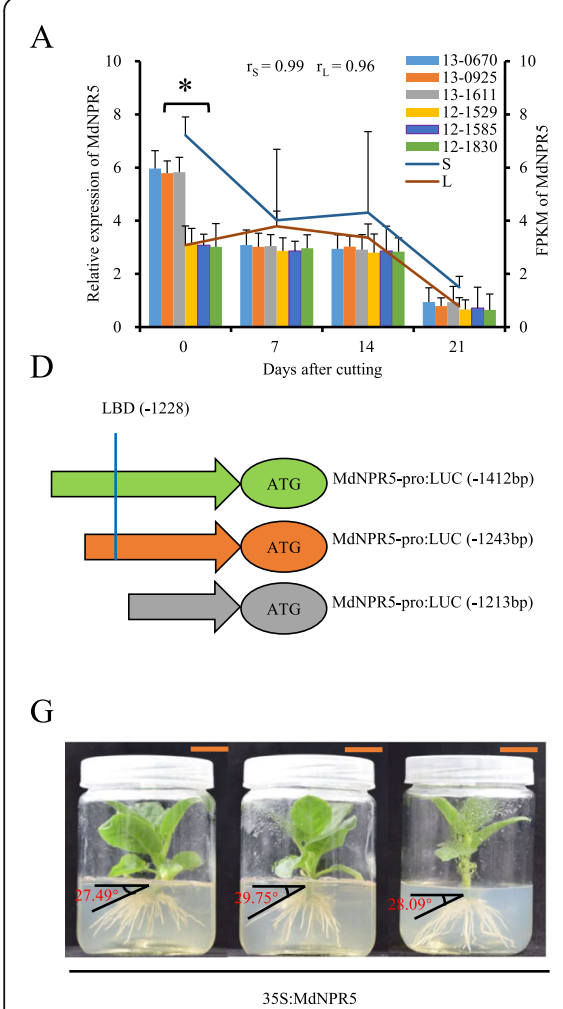

\section{B}

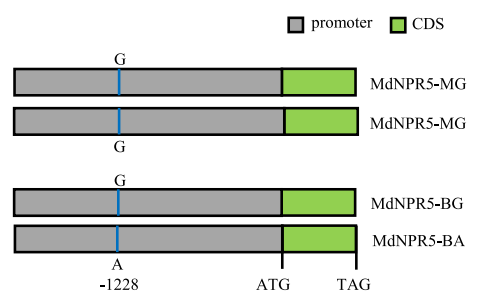

E
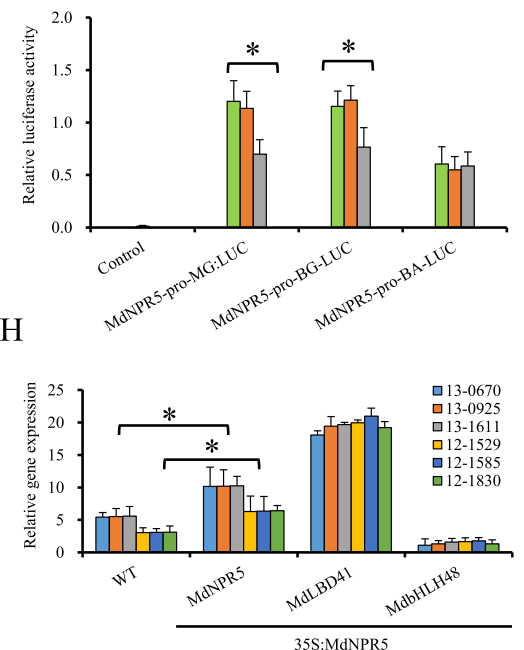

C

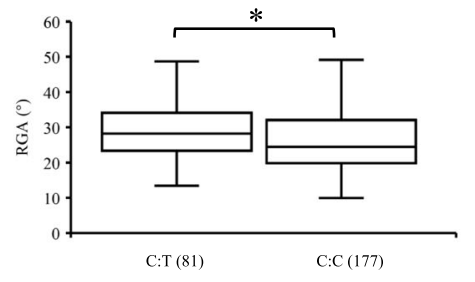

F

GFP

Merged Bright Field

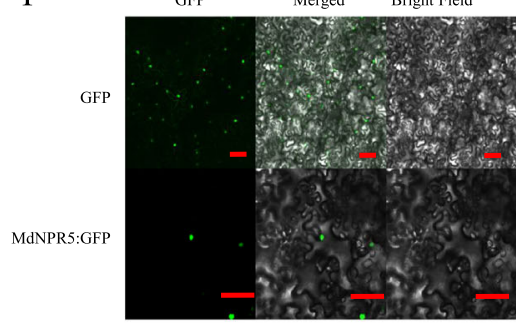

I

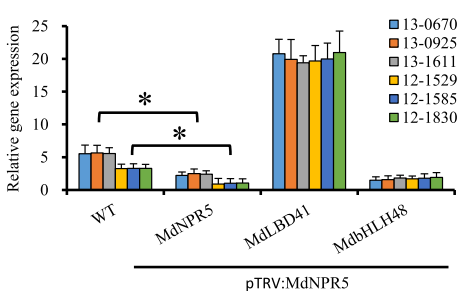

Fig. 4 Allelic variation of MdNPR5, effects on promoter activity, and function in adventitious RGA in 'BC', 'M9' and their F1 hybrids. a MdNPR5 expression by RT-qPCR (bar chart) and mean FPKM values (line chart) during adventitious root formation in hybrids with small (S) (13-0670, 130925, and 13-1611) and large (L) (12-1529, 12-1585, and 12-1830) RGAs. b Schematic representation of single nucleotide variation within the promoter of MdNPR5. c Box plots showing differences in RGA between hybrids with the C:T and C:C genotypes of marker S1272. Numbers of hybrids are presented in parentheses. $\mathbf{d}$ Schematic of promoter truncations of MdLNPR5-pro: LUC vector constructs with or without the LBD ciselement. e Transient expression analysis of various MdNPR5-pro: LUC constructs. "pro" represents the promoter. f Subcellular localization of transiently expressed MdNPR5:GFP in N. benthamiana leaves. Scale bars $=50 \mathrm{~mm}$. g RGA phenotypes of the 35S:MdNPR5 transgenic N. benthamiana lines and untransformed wild type. The RGA values are presented in parentheses. $\mathbf{h}$ and (i) Relative expression of MdLBD41 and MdbHLH48 when MdNPR5 was transiently expressed by the vectors 35S:MdNPR5 (h) and pTRV:MdNPR5 (i). Asterisks represent $\mathrm{P}<0.05$ by Dunnett's multiple comparison

small RGAs than in those with large RGAs at $0 \mathrm{~d}$ after cutting (Fig. 5a). The full-length CDS of MdLBD41 comprises two exons, which encode a 305-aa protein. SNP908 T/C caused an amino acid substitution. Another synonymous SNP713 A/C was homozygous in both parents and did not segregate in their F1 population (Fig. 5c; Table S11; Supplementary File 4A and B). The RGAs of 78 hybrids with the G:T genotype of marker Z312, which was linked with SNP908 T:C, were significantly larger than those of 186 hybrids with the Z312 G:G genotype, which was linked with SNP908 T:T (Fig. 5e; Table S12). The promoter activity of the $1655 \mathrm{bp}$ DNA sequences upstream in MdLBD41 was similar between 'BC' and 'M9' (Fig. 5g; Supplementary File 4C). These data indicated that a SNP908 variant (rather than variations of the promoter) affected MdLBD41 function.

The relative expression and FPKM values of MdbHLH48 were significantly higher at $\mathrm{d} 0$ and 21 after cutting in the stem tissues of hybrids with large RGAs than those with small RGAs (Fig. 5b). MdbHLH48 encoded a 357-aa protein and had five closely linked non-synonymous SNPs in the CDS between 'BC' and 'M9': SNP344 G/A, SNP644 A/C, SNP785 G/A, SNP991 A/G, and SNP1006 T/C. There was also an 11-bp deletion between -360 and -370 bp upstream of the ATG codon (Del360), which altered the GA-rich motif in 'BC' but not in 'M9' (Fig. 5d; Table S11; Supplementary File 5). The RGA of 135 hybrids with the S1288 C:A genotype, which was linked with Ins360:Del360, was significantly larger than that of 129 hybrids with the S1288 C:C genotype, which was linked with Ins360:Ins360, according to the KASP assay (Fig. 5f; Table S12). The MdbHLH48 promoter truncation assay showed that truncating the Ins360 allele in MIns360 and BIns360 led to a significant increase in LUC activity (Fig. $5 \mathrm{~h}$ and i). These data indicated that GA-rich elements had a negative effect on MdbHLH48 transcription and that Del360 completely destroyed the GA-rich element. 


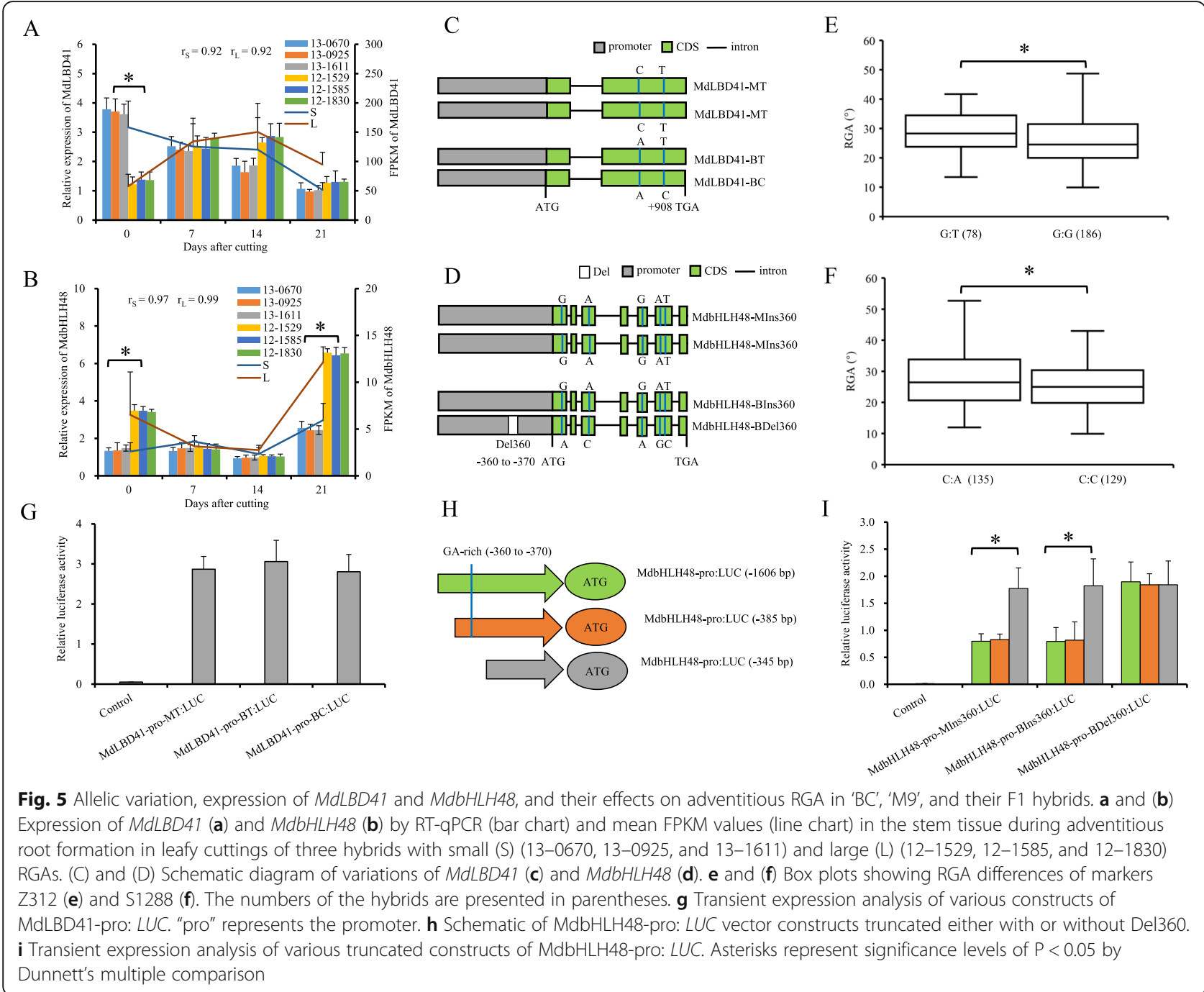

\section{Non-allelic interactions of MdNPR5, MdLBD41 and MdbHLH48}

The $\mathrm{Y} 1 \mathrm{H}$ assay showed that the protein variants MdLBD41-MT, MdLBD41-BT, and MdLBD41-BC interacted with the promoters of both MdNPR5-MG and MdNPR5-BG but not with MdNPR5-BA (Fig. 6a). Higher levels of LUC activity were detected in MdNPR5-pro-MG: LUC and in MdNPR5-pro-BG:LUC than in MdNPR5-proBA:LUC coinjected with any of 35S:MdLBD41 variants or in the non-coinjected control (Fig. 6b). These results indicated that $M d L B D 41$ positively regulated the expression of $M d N P R 5$ via the LBD cis-element.

MdbHLH48-MIns360 and MdbHLH48-BDel360 did not interact with MdNPR5, according to $\mathrm{Y} 1 \mathrm{H}$ assay (Fig. 6a). However, the $\mathrm{Y} 2 \mathrm{H}$ and BIFC assays showed that MdLBD41-MT and MdLBD41-BT (rather than MdLBD41BC) interacted with both MdbHLH48-MIns360 and MdbH LH48-BDel360 (Fig. 6c; Fig. S7C). This indicated that SNP908 T/C allelic variation of MdLBD41 in the C- terminus damaged the binding ability with MdbHLH48. The LUC activity was significantly lower when MdNPR5pro-MG:LUC or MdNPR5-pro-BG:LUC (but not MdNPR 5-pro-BA:LUC) were coinjected with MdbHLH48-BDel360 or MdbHLH48-MIns360 and MdLBD41-BC than with MdLBD41-BT or MdLBD41-MT. (Fig. 6d). These data further indicated that the lack of interaction between MdLBD41 SNP908 allele C and MdbHLH48 variants negatively affects the activity of the MdNPR5 promoter.

$M d N P R 5$ and $M d L B D 41$ were located within the same interval of QTL B09.1; thus, both loci showed a close linkage, and 19 of 256 hybrids were recombinants (Table S14). The joint genotype effects showed that the RGAs of hybrids with the genotypes MdNPR5 SNP-1228 G:G and MdLBD41 SNP908 T:T were smaller $\left(-2.21^{\circ}\right.$ and $1.18^{\circ}$ ) than those of hybrids with one or two heterozygous genotypes, irrespective of $\mathrm{MdbHLH} 48$ genotypes. The MdbHLH48 Ins360:Del360 genotype exerted a greater effect on RGA than Ins360:Ins360 (Fig. 6e, Table 

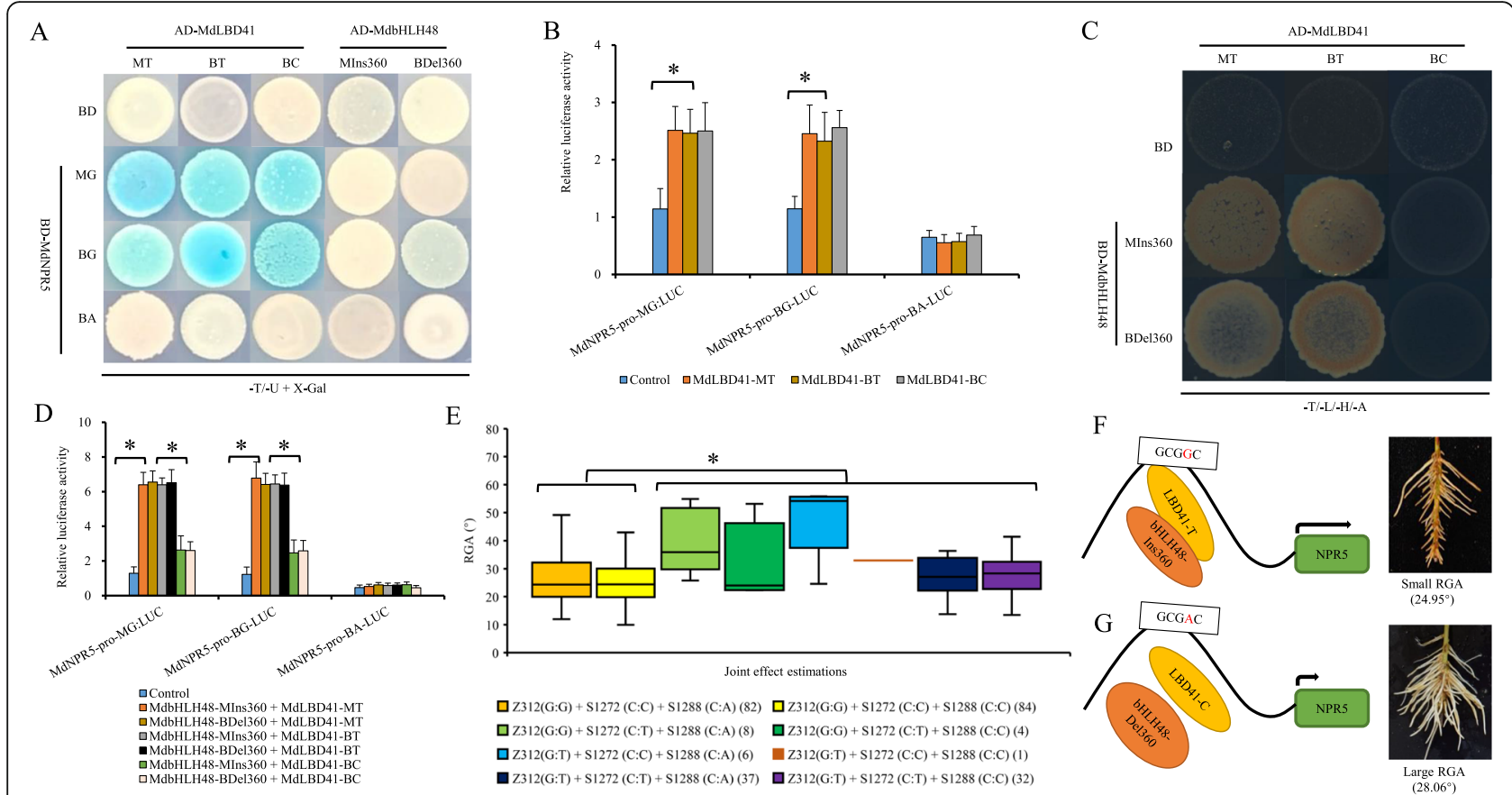

Fig. 6 Non-allelic interactions and joint effects of MdNPR5, MdLBD41, and MdbHLH48 natural variants on adventitious RGA in leafy cuttings of hybrids derived from 'BC' $\times$ ' $M 9^{\prime}$ apple rootstock. a Y1H image showing the interaction of the MdNPR5 promoter with MdLBD41 and MdbHLH48. b Transient coexpression assay of MdNPR5-pro: LUC variants interacting with 35S:MdLBD41 variants. c Y2H images showing interactions between variants of MdLBD41 and MdbHLH48. d Transient coexpression assay of MdNPR5-pro: LUC interacting with 35S:MdLBD41 and 35S:MdbHLH48 variants. e Joint genotype effect estimates of markers S1272 (C/T), Z312 (T/G), and S1288 (A/C). Numbers of the hybrids are presented in parentheses. Asterisks represent $\mathrm{P}<0.05$ by Dunnett's multiple comparison. $\mathbf{f}$ and $(\mathbf{g})$ Proposed model for the genetic variation networks regulating apple rootstock RGA by MdNPR5, MdLBD41, and MdbHLH48. The arrow length indicates the gene expression level. Numbers of the hybrids are presented in parentheses

S14). MdLBD41 and MdbHLH48 exhibited epistatic non-allelic effects on MdNPR5 (Fig. $6 \mathrm{f}$ and g).

\section{Marker genotype effect estimation}

To estimate the QTL-based marker effect on RGA, 26 SNPs were selected for the design of KASP markers. These SNPs were selected on candidate genes near the peak of the abovementioned 25 QTLs (Table S15). These included six diagnostic markers, SNP592, G122, b13, Z312, S1272, and S1288, the latter five of which are tightly linked to MdIAA1 SNP223, MdLAZY1 SNP1485/-474, MdLBD41 SNP908, MdNPR5 SNP-1228, and MdbHLH48 Del360 (Table S12). These markers were genotyped by KASP assay in 266 randomly chosen F1 hybrids from the 'BC' $\times$ 'M9' population (Table S16). Both the marker effects and the marker genotype effects varied considerably (Table S17). The marker Z1796 from QTL H04.1 exhibited the largest marker effect $\left(26.11^{\circ}\right)$ on RGA, whereas the marker effect $\left(0.22^{\circ}\right)$ of Z4196 from QTL M16.1 was the smallest (Table S17). The largest genotype effect was estimated for Z1796 A:A $\left(23.53^{\circ}\right)$, while the smallest genotype effect was detected for G122 C:A $\left(-4.00^{\circ}\right)$ (Table S17). Of these six diagnostic markers, SNP592 T:T and G122 A:A displayed the largest non-allelic epistatic effects $\left(6.14^{\circ}\right)$ on b13 T:G (Table S13). The smallest non-allelic effects $\left(-2.21^{\circ}\right)$ on S1272 C:C were clearly detected for markers Z312 G:G and S1288 C:C (Table S14).

\section{Discussion}

Adventitious RGA is controlled by multiple genes with varied effects in apple

The nodal root angle of sorghum measured on the 141 F6 recombinant inbred lines ranged from $14.5^{\circ}$ to $32.3^{\circ}$, and the heritability was $73.7 \%$ [60]. The seminal RGA of wheat ranged from $25^{\circ}$ to $60^{\circ}$ in 103 double haploid lines, and the estimated heritability was 0.43 [65]. In this study, the RGA of a population of 1955 hybrids from 'BC' $\times$ 'M9' segregated continuously with an overall deviation of $9.26-$ $58.95^{\circ}$ and broad-sense heritability of $85.26 \%$, which were consistent with those in sorghum and wheat and were similar to our previous findings [77]. Four to five QTLs for RGA were detected in maize, rice, and sorghum [7, 9, $60,63,64]$. In the current study, as many as 25 QTLs of RGA were identified in apple rootstock. Of the 25 QTLs for RGA in this study, 11,8 , and 6 were of the B, M, and $\mathrm{H}$ type, respectively, markers of which were heterozygous for the maternal parent, the pollen parent, and both 
parents, respectively. These data indicated that apple RGA is relatively less affected by the environment but has more complicated multi-genetic control than that observed in cereal crops.

Adventitious root formation from stem cuttings is usually divided into three stages based on physiological and metabolic markers and can be categorized as root induction, root initiation, and root extension [1]. The first critical stage of adventitious rooting regulation occurs during adventitious root induction [78, 79]. Data in this study showed that the earliest stage at which the RGA could be determined was before day 7 since up to $40 \%$ of DEGs were identified from transcriptomic data at day 0 . Moreover, most DEGs for auxin, cytokinin, gibberellin, and abscisic acid were detected at $0 \mathrm{~d}$, which suggests their important roles in the regulation of the RGA. Another root developmental process that determines RGA occurs during root elongation [80]. The adventitious roots in apple rootstock undergo elongation 14-21 $\mathrm{d}$ after cutting [81]. In this study, $44.12 \%$ of DEGs were detected at day 21 after cutting, indicating that RGA may also be associated with root elongation.

\section{Natural variations involving auxin signalling and gravitropism contributed to genetic diversity in the RGA} During root formation and development processes, auxin regulates the gravitropic set-point angles by adjusting the magnitude of the anti-gravitropic offset component via TIR1/AFB-Aux/IAA-ARF-dependent auxin signalling within the gravity-sensing cells of the roots [82]. Some functional genes, DRO1, LAZY1 and qSOR1, which regulate root gravitropism by auxin signalling, are closely associated with RGA [7, 41, 83]. In this study, 153 DEGs involved in gravitropism, auxin signalling, and related pathways were detected by RNA-seq. Similarly, from QTL intervals, candidate RGA genes (MdLAZY1, MdIAA1, MdDREB2A, MdNPR5, MdLBD41 and MdbHLH48) were also involved in auxin signalling and root gravitropism.

Natural variation of the RGA has been reported, but the genetic basis for this variation is largely unknown $[9,10,61$, 66]. Introgression lines in rice demonstrated that combinations of allelic variations in qSOR1 and DRO1 controlled the RGA in rice [83]. To date, little is known about genetic variation in apple RGAs. The results of the present study revealed that six functional genes developed two epistatic non-allelic variation networks regulating the RGA. One epistatic non-allelic network showed that SNP-1485/- 474 of the MdLAZY1 promoter significantly affected the expression level and RGA by altering DRE and HSF cis-elements, which interacted with MdDREB2A and MdHSFB3, respectively. Then, the SNP592-induced stop loss variation on $M d D R E B 2 A$ affected the interaction with MdHSFB3, which in turn significantly influenced $M d L A Z Y 1$ expression. The structural variation of the MdDREB2A protein affects its function, not its expression, which is consistent with a report on Robinia pseudoacacia [50]. IAA17 interacted with LAZY1, which affected the auxin response and gravitropism in maize [43]. A SNP in the IAA core sequence interrupted root gravitropism, and it remains unclear whether this affected the interaction with LAZY1 [43, 46-48]. In this study, SNP223 at the MdIAA1 CDS affected the interaction with MdLAZY1 and subsequently the RGA of cuttings.

Another epistatic non-allelic network controlling RGA was formed by SNP-1228 of MdNPR5, SNP908 of MdLBD41, and Del360 of MdbHLH48. SNP-1228 damaged the LBD cis-element of the MdNPR5 promoter, which affected the interaction with the MdLBD41 protein, as well as the expression of MdNPR5 and the RGA. SNP908 in the C-terminus of MdLBD41 also influenced the transcription activity of the target gene MdNPR5 by altering the protein-to-protein interaction between MdLBD41 and MdbHLH48 that has been reported for Arabidopsis [56]. The GA-rich cis-element exerts a negative regulatory effect on its own promoter [84]. Del360 of MdbHLH48 increased both the gene expression of MdbHLH48 and the RGA by affecting the GArich cis-element. Moreover, higher MdbHLH48 expression levels at $\mathrm{d} 0$ and 21 after cutting were not consistent with the lower levels of MdNPR5 expression, indicating the possible existence of an undiscovered regulatory mechanism.

\section{QTL-based markers will be a potentially efficient tool for the breeding of deep root architecture}

To date, many QTL mapping studies for diverse species have provided an abundance of DNA marker-trait associations $[85,86]$. To increase the selection efficiency and the predictability of genetic values, candidate genes are often predicted within the significant intervals of QTLs. The ideal marker can be designed to the functional variation loci of the candidate genes, which effectively eliminates the linkage disequilibrium decay of these QTL-based markers [87]. These markers are sometimes called diagnostic markers [88]. In this study, 26 QTL-based markers were developed, the marker effects varied widely from $0.22^{\circ}$ to $26.11^{\circ}$, and the marker genotype effect varied from $-4.00^{\circ}$ to $23.53^{\circ}$. Diagnostic markers SNP592, G122, b13, Z312, S1272, and S1288 were closely linked with functional variation loci by experimental validation.

The predictability of QTL-based markers is likely not transferable to different populations [89, 90]. However, the markers for RGA in this study can potentially be used for selecting materials that are genetically related to ' $\mathrm{M} 9$ ' and 'BC', because both 'M9' and 'BC' have been frequently used as parental cultivars in apple rootstock breeding programmes [91-94]. Apple dwarfing rootstocks usually have a smaller RGA and relatively shallow root architecture [2]. The QTLs for dwarfing ability, DW1, DW2, and DW3, 
have been successfully mapped in 'M9' apple rootstock [92-94]. None of the 25 QTLs for RGA in this study overlapped with the DW1/DW2/DW3 QTL regions, suggesting that RGA is related but not genetically linked to dwarfing ability in apple rootstocks. These data indicated that it will be practical to select apple rootstocks with both large RGAs for better anchorage and dwarfing ability for high-density planting.

\section{Conclusions}

A total of 25 significant QTLs were identified, 11 of which were mapped on the maternal parent ' $\mathrm{BC}$ ', eight were mapped on the pollen parent 'M9', and six were located on both parents. Transcriptomic data showed that the RGA was determined before $7 \mathrm{~d}$ and after $14 \mathrm{~d}$, which implied that the critical stage of RGA regulation occurs during initiation and elongation of adventitious roots. Six markers were linked to the exact loci of the functional variations of genes and were involved in two molecular regulatory pathways of adventitious RGA in apple. SNP223 of MdIAA1, SNP592 of MdDREB2A, and SNP-1485/- 474 of MdLAZY1 formed an epistatic non-allelic network. Furthermore, Del360 of MdbHLH48, SNP 1228 of MdNPR5, and SNP 908 of MdLBD41 formed another epistatic non-allelic network. A total of 26 QTL-based markers were developed with varied marker effects and genotype effects on RGA. These markers can potentially assist future breeding programmes aimed at optimizing the RGA in apple rootstocks.

\section{Methods}

\section{Plant materials and phenotyping}

All plant materials were obtained from China Agricultural University. The experimental research on plants, including field investigation and sample collection, was performed under institutional guidelines in accordance with local legislation. No formal identification was performed, and no voucher was deposited in a publicly available herbarium. During May 2016 and 2017, more than 30 semi-lignified leafy cuttings $(10 \mathrm{~cm}$ in length) were collected from each of the 1955 and $1383 \mathrm{~F} 1$ hybrids derived from 'M9' (small RGA) × 'BC' (large RGA), respectively. The basal end of the stem cuttings $(1 \mathrm{~cm})$ was immediately submerged in indole-3-butyric acid (IBA, $3000 \mu \mathrm{g} / \mathrm{L}$ ) for $1 \mathrm{~min}$ and plugged in trays filled with sand in a greenhouse. The relative air humidity was controlled at $95 \%$ using a fog irrigator. Thirty $d$ after cutting, cuttings with roots were collected, washed with tap water, and photographed. The RGAs were measured using an ImageJ 1.50 scanner (National Institutes of Health, Bethesda, MD, USA) [77]. Statistical analysis was performed using SPSS 17 software (Armonk, NY, USA).

\section{BSA-seq}

To obtain the two extreme bulks for BSA-seq, 30 hybrids with extremely large RGAs and 30 hybrids with extremely small RGAs were selected based on two-year phenotyping data. The genomic DNA of the selected hybrids was extracted from young leaves and pooled from equal quantities (i.e., $500 \mathrm{ng}$ each). The two bulked DNA samples were fragmented via sonication to $300-650 \mathrm{bp}$ for library construction and were paired-end sequenced using the Illumina HiSeq X-Ten platform (Illumina, San Diego, CA, USA). Then, Burrows-Wheeler Alignment (BWA) software was used to map the clean sequencing reads to the apple genome GDDH13 [95]. The default values were used for read mapping, and only uniquely mapped reads with a minimum phred score of 20 were retained [96]. SAMtool software was used to identify SNPs and indels between the aligned sequencing reads and the reference genome [97]. The G' value method was used to statistically analyse the allelic variations in the two extreme bulks [98]. QTLs were identified using BSA Tools for Outbreeding Species (BSATOS) software, and the QTL regions were further narrowed by using varying sliding windows [96]. Analysis of allelic variations in QTLs was performed three times (pollen, maternal, or both parents) using two parental resequencing data sets [96].

\section{RNA-seq analysis}

Three hybrids, as three biological replicates, were randomly chosen from each of the two extreme bulks and were subjected to RNA-seq analysis. At least five cuttings of each hybrid were sampled at $0 \mathrm{~d}, 7 \mathrm{~d}, 14 \mathrm{~d}$, and $21 \mathrm{~d}$ after cutting based on previously reported anatomical changes during the formation of adventitious roots $[1,81]$. Approximately $0.5 \mathrm{~cm}$ of basal stem tissue was sampled, including the adventitious root zone. Total RNA was extracted by using the CTAB method [99]. The mRNA of two RNA-seq libraries was isolated from $5 \mu \mathrm{g}$ of total RNA from each sample. Then, the NEBNext Poly (A) mRNA Magnetic Isolation Module and NEBNext Ultra Directional RNA Library Prep Kit for Illumina (New England Biolabs, Ipswich, MA, USA) were applied for the preparation of two RNA-seq libraries. The cDNA libraries were sequenced (paired-end 150) from the $5^{\prime}$ to the $3^{\prime}$ ends on the Illumina HiSeq X Ten platform (Illumina). Then, clean RNA-seq reads were mapped to the apple genome GDDH13 by HISAT2 software [100]. Transcript assembly and quantification were conducted by StringTie [101]. The DEGs between small and large RGAs were analysed by DESeq2 software [102]. GO classification and GO enrichment were analysed by Blast2GO [103]. The KEGG metabolism annotation and KEGG enrichment were analysed by KOBAS2.0 [104]. The DEG analysis of major KEGG pathways $(P<0.01)$ was assessed by online network analysis in AppleMDO [105]. 


\section{Candidate gene prediction from QTLs}

The genes from each QTL interval were downloaded from the apple genome GDDH13 (https://www.rosaceae. org/) [95]. By comparing parental resequencing data, genes with identified genetic variations on the parental cultivar on which the QTL was not mapped were removed from the list [96]. Genes that were not expressed throughout and genes with SNPs or SVs only within the promoter that did not show differential expression by RNA-seq were removed. For the remaining genes, the UniProt database (http://www.UniProtuniprot.org/) was used to assess the function of the corresponding proteins. Genes that were clearly not related to the target trait were excluded. The pipeline for candidate prediction has been published previously $[96,106]$.

\section{Validation and analysis of allelic variations}

The 2-kb promoter sequences and the full-length coding sequence were amplified and Sanger sequenced to validate predicted variations. The primers are listed in supplementary Table S18. Analyses of amino acid sequences were performed by DNAMAN8 software. Analyses of cis-elements were performed by the online tools Plant CARE and JASPAR [107].

\section{Gene expression analysis}

Total RNA was extracted from stem tissues of cuttings, and cDNA was synthesized. The relative expression of candidate genes was measured by real-time quantitative PCR (RT-qPCR) [107]. Analysis of variance and Dunnett's multiple comparison were conducted to identify differences in gene expression. MdEF1 $\alpha-\mathrm{F}$ and MdEF1 $\alpha-R$ were selected as quantification controls, and the primers are listed in supplementary Table S18.

\section{Plasmid construction and genetic transformation}

The truncated promoter/LUC fusion vectors (pGreenII 0800-LUC) of MdLAZY1 and MdNPR5 were constructed by digestion with Pst1 and EcoR1. The full-length cDNA of MdDREB2A, MdHSFB3, MdLBD41, and MdbHLH48 was fused with the overexpression vector (pGreenII 62SK) by digestion with EcoR1 and Kpn1. The vectors were introduced into Agrobacterium tumefaciens strain GV1301, and PCR was used to confirm their introduction. Then, the truncated promoter/LUC fusion vectors of MdLAZY1 and MdNPR5 were transformed into leaf epidermal cells of one-month-old $N$. benthamiana plants and an empty vector pGreenII 0800-LUC as a control. The fusion vectors of promoter/LUC and the overexpression vector were also coinjected into leaf epidermal cells of one-month $N$. benthamiana at a 1:1 ratio. Moreover, the coinjection of promoter/LUC fusion vectors and the empty overexpression vector were used as controls. The
LUC activities were measured with three biological replicates [108]. All primers are listed in Table S18.

The overexpression vectors (PRI101-AN) of MdLAZY1 and MdNPR5 were constructed by digestion with Kpn1 and EcoR1. The pTRV2 vectors of MdLAZY1 and $M d N$ PR5 were constructed by digestion with Kpn1 and Xho1. Then, 35S:MdLAZY1, 35S:NPR5, and empty vectors were introduced into $N$. benthamiana by $A$. tumefaciens-mediated transformation [81]. The RGA of transgenic lines and the WT were measured at $14 \mathrm{~d}$ after subculture using an ImageJ 1.50 scanner. The 35S:MdLAZY1, 35S:NPR5, pTRV2:MdLAZY1, pTRV2:MdNPR5, and empty vectors were transformed into each of the three hybrids chosen from both extreme bulks via vacuum infiltration [109]. Identifications of transformation lines by RT-qPCR and PCR were performed as described above, and the primers are listed in Table S18.

\section{Subcellular localization of MdLAZY1 and MdNPR5}

The full-length cDNAs of MdLAZY1 and MdNPR5 (without stop codons) were fused with PRI101-GFP by digestion with Kpn1 and BamHI. The two constructed vectors were introduced into $A$. tumefaciens strain GV1301 and were transformed into leaf epidermal cells of one-month-old $N$. benthamiana plants and visualized using confocal microscopy [110]. The control was obtained by treating tobacco leaves with the empty vectors of PRI101-GFP. All primers are listed in Table S18.

\section{$\mathrm{Y} 1 \mathrm{H}$ assay}

The full-length cDNAs of different genotypes of MdDREB2A, MdHSFB3, MdbHLH48, and MdLBD41 were fused with $A D$ vectors (PJG4-5) by digestion with EcoR1 and Xho1. Furthermore, the promoters from different genotypes of MdLAZY1 and MdNPR5 were fused with $\mathrm{BD}$ vectors (Placzi) by digestion with EcoR1 and $\mathrm{Xho1}$, respectively. The vectors with $\mathrm{AD}$ and $\mathrm{BD}$ were cotransformed into the $\mathrm{Y} 1 \mathrm{H}$ yeast strain on SD medium without Trp and Ura $(-\mathrm{T} /-\mathrm{U})$ at $30^{\circ} \mathrm{C}$ for 3 $\mathrm{d}$. Then, the successful transformants were selected and grown on the previous $\mathrm{SD}$ medium with $\mathrm{X}$-gal $(-\mathrm{T} /-\mathrm{U} / \mathrm{X}$-gal $)$ at $30^{\circ} \mathrm{C}$ for $3 \mathrm{~d}$. The primers are listed in Table S18.

\section{$\mathrm{Y} 2 \mathrm{H}$ assay}

The full-length cDNAs of different genotypes from $M d D R E B 2 A, M d b H L H 48$, and MdLAZY1 were fused with BD vectors (pGBKT7) by digestion with EcoR1 and Sal1. At the same time, the full-length cDNAs of different genotypes from MdLBD41, MdIAA1, and MdHSFB3 were fused with AD vectors (pGADT7) by digestion with EcoR1 and Xho1, respectively. The vectors with $\mathrm{AD}$ and $\mathrm{BD}$ were cotransformed into the $\mathrm{Y} 2 \mathrm{H}$ yeast strain on $\mathrm{SD}$ 
medium without Leu and $\operatorname{Trp}(-\mathrm{L} /-\mathrm{T})$ at $30^{\circ} \mathrm{C}$ for $3 \mathrm{~d}$. Then, successful transformants were selected and grown on SD medium without Leu, Trp, His, and Ade (-L/-T/ $-\mathrm{H}-\mathrm{A})$ at $30^{\circ} \mathrm{C}$ for $3-5 \mathrm{~d}$. The primers are listed in Table S18.

\section{BiFC assay}

MdDREB2A, MdbHLH48, and MdLAZY1 without stop codons in the coding sequence were cloned into NE vectors (SPYNE) by digestion with $\mathrm{XbaI}$ and BamHI. $M d L B D 41, M d I A A 1$, and MdHSFB3 without stop codons in the coding sequence were cloned into $\mathrm{CE}$ vectors (SPYCE) by digestion with XbaI and BamHI. The fusion plasmids were cotransformed into $N$. benthamiana leaves, and transformed leaf epidermal cells were observed by confocal microscopy [111].

\section{Marker genotype effect estimation by KASP assay}

KASP primers were designed based on the 100-bp flanking sequences of the SNPs of candidate genes. Detailed protocols can be found in the KASP genotyping chemistry user guide and manual (http://www.lgcgenomics.com). The genomic DNAs of 266 randomly chosen hybrids from ' $\mathrm{BC}$ ' $\times$ ' M9' were extracted, and all the abovementioned markers were genotyped using the KASP assay (LGC Genomics, Beverly, MA, USA). Fluorescence detection was conducted by using an Omega Fluorostar scanner (BMG PHERAstar, BMG LabTech, Ortenberg, Germany). The genotype data were output by 'SNP VIEWER' software (LGC). SNP-1485/ - 474 of MdLAZY1, SNP223 of MdIAA1, SNP-1228 of MdNPR5, SNP908 of MdLBD41, and Del360 of MdbH LH48 were sequenced by PCR to measure how close these are linked with b13, G122, S1272, Z312, and S1288. The primers are listed in Table S18. The marker genotype effects were calculated by subtracting the average RGA phenotype value of a subset of hybrids with the same genotype from the overall mean phenotype value of the complete population. The marker effects were estimated using the genotype value deviation of a specific marker.

\section{Statistical analysis}

Differences between the control and experimental treatments were analysed by one-way analysis of variance (ANOVA) through Dunnett's multiple comparison at a significance level of $\alpha=0.05$.

\section{Supplementary Information}

The online version contains supplementary material available at https://doi. org/10.1186/s12864-020-07257-8.

Additional file 1: Fig. S1 Segregation and segregant bulk construction of the adventitious root growth angle (RGA) in leafy cuttings of a hybrid population derived from 'Baleng $\mathrm{Crab}(\mathrm{BC})$ ' (Malus robusta) $\times$ 'M9' (M. pumila) in 2016 and 2017. (A) and (B) Frequency distribution diagrams of RGA in 2016 (A) and 2017 (B). (C) Photographs of adventitious roots in 30-day-old leafy cuttings, showing small and large RGAs. Scale bar $=10$ $\mathrm{mm}$. (D) Box plots showing the RGA phenotype in the small (left) and large (right) segregant bulks. Numbers of hybrids are presented in parentheses following the genotypes below the $x$-axis. Error bars indicate the standard deviation, and asterisks represent $P<0.05$ by Dunnett's multiple comparison.

Additional file 2: Fig. S2 Profiles of the significant quantitative trait loci (QTLs) for adventitious RGA in a hybrid from $B C^{\prime} \times{ }^{\prime} M 9^{\prime}$. The $y$-axis represents the $G$ ' value, and the $x$-axis represents the physical position on the chromosome. Red lines represent ' $M 9$ ', blue lines represent ' $\mathrm{BC}$ ', and black lines represent ' $M 9$ ' \& 'BC'.

Additional file 3: Fig. S3 Differentially expressed unigenes (DEGs) in the extreme small and large RGA bulks in a hybrid from ' $B C^{\prime} \times{ }^{\prime} M 9^{\prime}$. (A) Statistical data of DEGs. (B-E) Kyoto Encyclopedia of Genes and Genomes (KEGG) pathway results for including plant hormone signalling (B), starch and sucrose metabolism (C), terpenoid backbone biosynthesis (D), and alpha-linolenic acid metabolism (E).

Additional file 4: Fig. S4 Gene ontology (GO) analysis of differentially expressed genes (DEGs) from the RNA sequencing (RNA-seq) analysis. (A) GO classification. (B) GO enrichment. (C) GO statistics.

Additional file 5: Fig. S5 Kyoto Encyclopedia of Genes and Genomes (KEGG) analysis of RNA-seq results. (A) KEGG classification. (B) KEGG statistics. (C) KEGG enrichment. (D) KEGG screening.

Additional file 6: Fig. S6 Validation of 35S:MdLAZY1 (A) and 35S:MdNPR5 (B) transgenic Nicotiana benthamiana lines at the DNA (left) and CDNA (right) levels.

Additional file 7: Fig. S7 Bimolecular fluorescence complementation (BiFC) assay showing protein-protein interactions. (A) MdLAZY1 and MdIAA1. (B) MdDREB2A and MdHSFB3. BD-DREB2A-BC ( $p)$ indicates a point mutation of C (SNP592) to T in MdDREB2A-BC. (C) MdLBD41 and MdbHLH48. Scale bars $=50 \mathrm{~mm}$.

Additional file 8: Table S1 The phenotype data for the adventitious root growth angle (RGA) of leafy cuttings of hybrids derived from 'Baleng Crab (BC)' (Malus robusta) X'M9' (M. pumila) in 2016 and 2017. Table S2 Summary of the results of bulked segregant analysis by next generation genomic sequencing (BSA-seq) of extreme bulks of small and large adventitious RGAs using hybrids derived from ' $B C^{\prime} \times{ }^{\prime} M 9^{\prime}$. Table S3 Summary of BSA-seq detected QTLs for apple adventitious RGA before and after narrowing by changing slide window sizes. Table S4 Twentyfour stem tissue samples for RNA sequencing (RNA-seq) taken from leafy cuttings of hybrids derived from ' $B C^{\prime} \times{ }^{\prime} M 9^{\prime}$. Table S5 Summary of the sequencing reads and reads mapped with RNA-seq. Table S6 Sample correlation analysis of three biological replicates with RNA-seq. Table S7 KEGG pathway analysis of DEGs (ko00500, ko00592, ko00900, and ko04075) from RNA-seq results. Table S8 Co-expression network of RGA identified by AppleMDO in hybrids from 'BC' $\times$ 'M9'. Table $\mathbf{S 9}$ Candidate genes for RGA screened by parental resequencing, BSA-seq, and RNA-seq in a hybrid population from 'BC' $\times{ }^{\prime} M 9^{\prime}$. Table $\mathbf{S 1 0}$ Candidate gene mining from BSA-seq regions based on multiomics data. Table S11 Allelic variations of MdLAZY1, MdIAA1, MdDREB2A, MdHSFB3, MdNPR5, MdLBD41, and MdbHLH48. Table $\mathbf{S 1 2}$ Closely linked relationship between six diagnostic markers (SNP-1485/- 474 of MdLAZY1, SNP223 of MdIAA1, SNP1228 of MdNPR5, SNP908 of MdLBD41, and Del360 of MdbHLH48) and Kompetitive Allele Specific PCR (KASP) markers (b13, G122, S1272, Z312, and S1288). Table S13 Joint genotype effects of markers linked to b13, SNP592, and G122 on RGA phenotype in hybrids from 'BC' $\times$ ' $M 9$ '. Table S14 Joint genotype effects of markers linked to S1272, Z312, and S1288 on RGA phenotype in hybrids from 'BC' $\times$ 'M9'. Table S15 Detailed data and primers for 26 allelic variations used for KASP in 'BC' and ' $M 9$ '. Table S16 The genotypes of 26 markers in 266 F1 hybrids from ' $B C^{\prime} \times{ }^{\prime} M 9$ '. Table S17 The individual genotype effects of the 26 markers in 266 F1 hybrids from 'BC' $\times$ 'M9'. Table $\mathbf{S 1 8}$ Primers used for experimental validation in this study.

Additional file 9: Supplementary File 1 Sequence alignment of MdLAZY1 cloned from apple rootstocks 'Baleng Crab (BC)' (Malus robusta) 
and 'M9' (M. pumila). (A) Coding sequence (CDS). (B) Amino acid. C: Upstream.

Additional file 10: Supplementary File 2 Sequence alignment of MdIAAI, MdDREB2A, and MdHSFB3 cloned from apple rootstocks 'BC' and 'M9'. (A), (C), and (E) CDS alignment of MdIAAT (A), MdDREB2A (C), and MdHSFB3 (E), respectively. (B), (D), and (F) Amino acid sequences of $\operatorname{MdIAA1}(\mathrm{B}), \operatorname{MdDREB2A}(\mathrm{D})$, and MdHSFB3 (F), respectively.

Additional file 11: Supplementary File 3 Sequence alignment of MdNPR5 cloned from apple rootstocks 'BC' and 'M9'. (A) CDS. (B) Amino acid. (C) Upstream.

Additional file 12: Supplementary File 4 Sequence alignment of MdLBD41 cloned from apple rootstocks 'BC' and 'M9'. (A) CDS. (B) Amino acid. (C) Upstream.

Additional file 13: Supplementary File 5 Sequence alignment of MdbHLH48 cloned from apple rootstocks 'BC' and 'M9'. (A) CDS. (B) Amino acid. (C) Upstream.

\section{Abbreviations}

BiFC: Bimolecular fluorescence complementation; BSA-seq: bulked segregant analysis by sequencing; DEG: Differentially expressed unigene; KEGG: Kyoto Encyclopedia of Genes and Genomes; FPKM: Fragments per kilobase per million mapped reads; GO: Gene ontology; GS: Genomic selection; KASP: Kompetitive allele specific PCR; LUC: Luciferase; MAS: Marker-assisted selection; QTL: Quantitative trait locus; RGA: Root growth angle; RNAseq: RNA sequencing; SNP: Single nucleotide polymorphism; WT: Wild type: Y1H: Yeast one-hybrid; Y2H: Yeast two-hybrid

\section{Acknowledgements}

We thank the Key Laboratory of Biology and Genetic Improvement of Horticultural Crops (Nutrition and Physiology) of the Ministry of Agriculture, P. R. China, for providing the experimental platform.

\section{Authors' contributions}

C.Z., X.Z., and Z. H conceived and designed the experiments. Y.W., T.W., X.X., $X . Z$., and Z. H contributed plant materials. C. Z performed the experiments. F.S. performed the bioinformatic analysis. C.Z. and X.Z. wrote the paper. All authors have read and approved the manuscript.

\section{Funding}

This work was funded by the earmarked fund for the National Science Foundation (31772262), the Modern Agricultural Industry Technology System (CARS-27), the Beijing Municipal Education Commission (CEFFPXM2019_014207_000099), and the Construction of Beijing Science and Technology Innovation and Service Capacity in Top Subjects (CEFFPXM2019_014207_000032). The funding bodies had no role in the study design, data collection and analysis, decision to publish, or preparation of the manuscript.

\section{Availability of data and materials}

All RNA-seq reads and BSA-seq data have been deposited in the NCBI SRA under the accession number PRJNA655783 (https://www.ncbi.nlm.nih.gov/ search/all/?term=PRJNA655783). The DNA resequencing reads of 'Baleng Crab' and ' $M 9$ ' are freely available in the NCBI SRA under the accession numbers SRR12234087 (https:/www.ncbi.nlm.nih.gov/search/all/?term=SRR12234 087) and SRR12233711 (https://www.ncbi.nlm.nih.gov/search/all/?term= SRR12233711), respectively. The apple genome used was a version of the Malus $\times$ domestica genome GDDH13_v1.1 (GDDH13, https://iris.angers.inra.fr/ gddh13/).

\section{Ethics approval and consent to participate} Not applicable.

\section{Consent for publication}

Not applicable.

\section{Competing interests}

The authors declare that they have no competing interests.
Received: 11 March 2020 Accepted: 19 November 2020

Published online: 01 December 2020

\section{References}

1. Li K, Liang Y, Xing L, Mao J, Liu Z, Dong F, et al. Transcriptome analysis reveals multiple hormones wounding and sugar signaling pathways mediate adventitious root formation in apple rootstock. Int J Mol Sci. 2018; 19:2201. https://doi.org/10.3390/ijms19082201.

2. Ma L, Hou CW, Zhang XZ, Li HL, Han DG, Wang Y, et al. Seasonal growth and spatial distribution of apple tree roots on different rootstocks or interstems. J Am Soc Hortic Sci. 2013:138:79-87. https://doi.org/10.21273/ JASHS.138.2.79.

3. Kondo M, Pablico PP, Aragones DV, Agbisit R, Abe J, Morita S, et al. Genotypic and environmental variations in root morphology in rice genotypes under upland field conditions. Plant Soil. 2003;255:189-200. https://doi.org/10.1023/A:1026142904714.

4. Guseman JM, Webb K, Srinivasan C, Dardick C. DRO1 influences root system architecture in Arabidopsis and Prunus species. Plant J. 2017;89:1093-105. https://doi.org/10.1111/tpj.13470.

5. Maeght JL, Rewald B, Pierret A. How to study deep roots-and why it matters. Front Plant Sci. 2013;4:299. https://doi.org/10.3389/fpls.2013.00299.

6. Araki H, Morita S, Tatsumi J, lijima M. Physiol-morphological analysis on axile root growth in upland rice. Plant Prod Sci. 2002;5:286-93. https://doi.org/10. 1626/pps.5.286.

7. Uga Y, Sugimoto K, Ogawa S, Rane J, Ishitani M, Hara N, et al. Control of root system architecture by DEEPER ROOTING 1 increases rice yield under drought conditions. Nat Genet. 2013;45:1097-102. https://doi.org/10.1038/ ng.2725.

8. Kitomi Y, Kanno N, Kawai S, Mizubayashi T, Fukuoka S, Uga Y. QTLs underlying natural variation of root growth angle among rice cultivars with the same functional allele of DEEPER ROOTING 1. Rice. 2015;8:16. https://doi. org/10.1186/s12284-015-0049-2.

9. Omori F, Mano Y. QTL mapping of root angle in F2 populations from maize 'B73' × teosinte 'Zea luxurians'. Plant Root. 2007;1:57-65. https://doi.org/10. 3117/plantroot.1.57.

10. Lopez JR, Erickson JE, Munoz P, Saballos A, Felderhoff TJ, Vermerris W. QTLS associated with crown root angle stomatal conductance and maturity in sorghum. Plant Genome. 2017;10(2). https://doi.org/10.3835/ plantgenome2016.04.0038.

11. Oyanagi A, Nakamoto T, Morita S. The gravitropic response of roots and the shaping of the root system in cereal plants. Environ Exp Bot. 1993:33:14158. https://doi.org/10.1016/0098-8472(93)90062-K.

12. Chen RJ, Rosen E, Masson PH. Gravitropism in higher plants. Plant Physiol. 1999:120:343-50. https://doi.org/10.1104/pp.120.2.343.

13. Su SH, Gibbs NM, Jancewicz AL, Masson PH. Molecular mechanisms of root gravitropism. Curr Biol. 2017;27:964-72. https://doi.org/10.1016/j.cub.2017.07. 015.

14. Wolverton C, Mullen JL, Ishikawa H, Evans ML. Root gravitropism in response to a signal originating outside of the cap. Planta. 2002;215:153-7. https://doi.org/10.1007/s00425-001-0726-9.

15. Tsugeki R, Fedoroff NV. Genetic ablation of root cap cells in Arabidopsis. Proc Natl Acad Sci U S A. 1999;96:12941-6. https://doi.org/10.1073/pnas.96. 22.12941.

16. Leitz G, Kang BH, Schoenwaelder ME, Staehelin LA. Statolith sedimentation kinetics and force transduction to the cortical endoplasmic reticulum in gravity-sensing Arabidopsis columella cells. Plant Cell. 2009;21:843-60. https://doi.org/10.1105/tpc.108.065052.

17. Lin C, Sauter M. Control of adventitious root architecture in rice by darkness, light, and gravity. Plant Physiol. 2018;176:1352-64. https://doi.org/10.1104/ pp.17.01540

18. Sakai T, Kagawa T, Kasahara M, Swartz TE, Christie JM, Briggs WR, et al. Arabidopsis $\mathrm{nph} 1$ and $\mathrm{npl1}$, blue light receptors that mediate both phototropism and chloroplast relocation. Proc Natl Acad Sci U S A. 2001;98: 6969-74. https://doi.org/10.1073/pnas.101137598.

19. Kiss JZ, Mullen JL, Correll MJ, Hangarter RP. Phytochromes a and B mediate red-light-induced positive phototropism in roots. Plant Physiol. 2003;131: 1411-7. https://doi.org/10.1104/pp.013847.

20. Han IS, Tseng TS, Eisinger W, Briggs WR. Phytochrome a regulates the intracellular distribution of phototropin 1-green fluorescent protein in Arabidopsis thaliana. Plant Cell. 2008;20:2835-47. https://doi.org/10.1105/tpc. 108.059915 
21. Saucedo M, Ponce G, Campos ME, Eapen D, Garcia E, Lujan R, et al. An altered hydrotropic response (ahr1) mutant of Arabidopsis recovers root hydrotropism with cytokinin. J Exp Bot. 2012;63:3587-601. https://doi.org/10. 1093/jxb/ers025.

22. Antoni R, Dietrich D, Bennett MJ, Rodriguez PL. Hydrotropism: analysis of the root response to a moisture gradient. Methods Mol Biol. 2016;1398:3-9. https://doi.org/10.1007/978-1-4939-3356-3_1

23. Eapen D, Barroso ML, Campos ME, Ponce G, Corkidi G, Dubrovsky JG, et al. A no hydrotropic response root mutant that responds positively to gravitropism in Arabidopsis. Plant Physiol. 2003;131:536-46. https://doi.org/ 10.1104/pp.011841.

24. Kobayashi A, Takahashi A, Kakimoto Y, Miyazawa Y, Fujii N, Higashitani A, et al. A gene essential for hydrotropism in roots. Proc Natl Acad Sci U S A. 2007;104:4724-9. https://doi.org/10.1073/pnas.0609929104.

25. Miyazawa Y, Takahashi A, Kobayashi A, Kaneyasu T, Fujii N, Takahashi H. GNOM-mediated vesicular trafficking plays an essential role in hydrotropism of Arabidopsis roots. Plant Physiol. 2009;149:835-40. https://doi.org/10.1104/ pp.108.131003.

26. Moriwaki T, Miyazawa Y, Fujii N, Takahashi H. Light and abscisic acid signaling are integrated by MIZ1 gene expression and regulate hydrotropic response in roots of Arabidopsis thaliana. Plant Cell Environ. 2012;35:135968. https://doi.org/10.1111/j.1365-3040.2012.02493.x.

27. Ishikawa $H$, Evans ML. The role of the distal elongation zone in the response of maize roots to auxin and gravity. Plant Physiol. 1993;102:1203-10. https:// doi.org/10.1104/pp.102.4.1203.

28. Feraru MI, Kleine-Vehn J, Feraru E. Auxin carrier and signaling dynamics during gravitropic root growth. Methods Mol Biol. 2015;1309:71-80. https:// doi.org/10.1007/978-1-4939-2697-8_7.

29. Muller A, Guan C, Galweiler L, Tanzler P, Huijser P, Marchant A, et al. AtPIN2 defines a locus of Arabidopsis for root gravitropism control. EMBO J. 1998;17: 6903-11. https://doi.org/10.1093/emboj/17.23.6903.

30. Friml J, Wisniewska J, Benkova E, Mendgen K, Palme K. Lateral relocation of auxin efflux regulator PIN3 mediates tropism in Arabidopsis. Nature. 2002; 415:806-9. https://doi.org/10.1038/415806a.

31. Swarup R, Kramer EM, Perry P, Knox K, Leyser HM, Haseloff J, et al. Root gravitropism requires lateral root cap and epidermal cells for transport and response to a mobile auxin signal. Nat Cell Biol. 2005;7:1057-65. https://doi. org/10.1038/ncb1316.

32. Kleine-Vehn J, Ding Z, Jones AR, Tasaka M, Morita MT, Friml J. Gravityinduced PIN transcytosis for polarization of auxin fluxes in gravity-sensing root cells. Proc Natl Acad Sci U S A. 2010;107:22344-9. https://doi.org/10. 1073/pnas.1013145107.

33. Eysholdt DE, Sauter M. Root bending is antagonistically affected by hypoxia and ERF-mediated transcription via auxin signaling. Plant Physiol. 2017;175: 412-23. https://doi.org/10.1104/pp.17.00555.

34. Fukaki H, Tameda S, Masuda H, Tasaka M. Lateral root formation is blocked by a gain-of-function mutation in the SOLITARY-ROOT/IAA14 gene of Arabidopsis. Plant J. 2002;29:153-68. https://doi.org/10.1046/j.0960-7412.2001. 01201.x.

35. Nagpal P, Walker LM, Young JC, Sonawala A, Timpte C, Estelle M, et al. AXR2 encodes a member of the aux/IAA protein family. Plant Physiol. 2000;123: 563-74. https://doi.org/10.1104/pp.123.2.563.

36. Yang X, Lee S, So JH, Dharmasiri S, Dharmasiri N, Ge L, et al. The IAA1 protein is encoded by AXR5 and is a substrate of SCFTIR1. Plant J. 2004;40: 772-82. https://doi.org/10.1111/j.1365-313X.2004.02254.x.

37. Band LR, Wells DM, Larrieu A, Sun J, Middleton AM, French AP, et al. Root gravitropism is regulated by a transient lateral auxin gradient controlled by a tipping-point mechanism. Proc Natl Acad Sci U S A. 2012;109:4668-73. https://doi.org/10.1073/pnas.1201498109.

38. Li P, Wang Y, Qian Q, Fu Z, Wang M, Zeng D, et al. LAZY1 controls rice shoot gravitropism through regulating polar auxin transport. Cell Res. 2007; 17:402-10. https://doi.org/10.1038/cr.2007.38.

39. Yoshihara $T$, lino $M$. Identification of the gravitropism-related rice gene LAZY1 and elucidation of LAZY1-dependent and -independent gravity signaling pathways. Plant Cell Physiol. 2007:48:678-88. https://doi.org/10. 1093/pcp/pcm042

40. Yoshihara T, Spalding EP, lino M. AtLAZY1 is a signaling component required for gravitropism of the Arabidopsis thaliana inflorescence. Plant J. 2013;74: 267-79. https://doi.org/10.1111/tpj.12118.

41. Taniguchi M, Furutani M, Nishimura T, Nakamura M, Fushita T, lijima K, et al. The Arabidopsis LAZY1 family plays a key role in gravity signaling within statocytes and in branch angle control of roots and shoots. Plant Cell. 2017; 9:1984-99. https://doi.org/10.1105/tpc.16.00575.

42. Yoshihara T, Spalding EP. LAZY genes mediate the effects of gravity on auxin gradients and plant architecture. Plant Physiol. 2017;175:959-69. https://doi.org/10.1104/pp.17.00942.

43. Dong $Z$, Jiang $C$, Chen $X$, Zhang T, Ding L, Song W, et al. Maize LAZY mediates shoot gravitropism and inflorescence development through regulating auxin transport auxin signaling and light response. Plant Physiol. 2013;163:1306-22. https://doi.org/10.1104/pp.113.227314.

44. Zhang $\mathrm{N}, \mathrm{Yu} \mathrm{H}, \mathrm{Yu} \mathrm{H}$, Cai $Y$, Huang $\mathrm{L}$, Xu C, et al. A core regulatory pathway controlling rice tiller angle mediated by the $L A Z Y 1$-dependent asymmetric distribution of auxin. Plant Cell. 2018;30:1461-75. https://doi.org/10.1105/tpc 18.00063 .

45. Li Z, Liang Y, Yuan Y, Wang L, Meng X, Xiong G, et al. OsBRXL4 regulates shoot gravitropism and rice tiller angle through affecting LAZY1 nuclear localization. Mol Plant. 2019;12:1143-56. https://doi.org/10.1016/j.molp.2019. 05.014 .

46. Ni J, Wang GH, Zhu ZX, Zhang HH, Wu YR, Wu P. OsIAA23-mediated auxin signaling defines postembryonic maintenance of QC in rice. Plant J. 2011;68: 433-42. https://doi.org/10.1111/j.1365-313X.2011.04698.X.

47. Kitomi $Y$, Inahashi $H$, Takehisa $H$, Sato $Y$, Inukai $Y$. OsIAA13-mediated auxin signaling is involved in lateral root initiation in rice. Plant Sci. 2012;190:11622. https://doi.org/10.1016/j.plantsci.2012.04.005.

48. Zhu ZX, Liu Y, Liu SJ, Mao CZ, Wu YR, Wu P. A gain-of-function mutation in OsIAA11 affects lateral root development in rice. Mol Plant. 2012;5:154-61. https://doi.org/10.1093/mp/ssr074.

49. Sakuma Y, Liu Q, Dubouzet JG, Abe H, Shinozaki K, Yamaguchi-Shinozaki K. DNA-binding specificity of the ERF/AP2 domain of Arabidopsis DREBs transcription factors involved in dehydration- and cold-inducible gene expression. Biochem Biophys Res Commun. 2002;290:998-1009. https://doi. org/10.1006/bbrc.2001.6299.

50. Xiu Y, labal A, Zhu C, Wu G, Chang Y, Li N, et al. Improvement and transcriptome analysis of root architecture by overexpression of Fraxinus pennsylvanica DREB2A transcription factor in Robinia pseudoacacia L. 'Idaho'. Plant Biotechnol J. 2016;14:1456-69. https://doi.org/10.1111/pbi.12509.

51. Liu H, Wang $S, Y u X, Y u$ J, He X, Zhang $S$, et al. ARL1 a LOB-domain protein required for adventitious root formation in rice. Plant J. 2005:43:47-56. https://doi.org/10.1111/j.1365-313X.2005.02434.x.

52. Taramino G, Sauer M, Stauffer JL, Jr Multani D, Niu X, Sakai H, et al. The maize (Zea mays L.) RTCS gene encodes a LOB domain protein that is a key regulator of embryonic seminal and post-embryonic shoot-borne root initiation. Plant J. 2007;50:649-59. https://doi.org/10.1111/j.1365-313X.2007. 03075.x.

53. Xu C, Tai H, Saleem M, Ludwig Y, Majer C, Berendzen KW, et al. Cooperative action of the paralogous maize lateral organ boundaries (LOB) domain proteins RTCS and RTCL in shoot-borne root formation. New Phytol. 2015; 207:1123-33. https://doi.org/10.1111/nph.13420.

54. Norberg $M$, Holmlund $M$, Nilsson O. The BLADE ON PETIOLE genes act redundantly to control the growth and development of lateral organs. Development. 2005;132:2203-13. https://doi.org/10.1242/dev.01815.

55. Ha CM, Jun JH, Nam HG, Fletcher JC. BLADE-ON-PETIOLE1 and 2 control Arabidopsis lateral organ fate through regulation of $L O B$ domain and adaxial-abaxial polarity genes. Plant Cell. 2007;19:1809-25. https://doi.org/10. 1105/tpc.107.051938.

56. Husbands A, Bell EM, Shuai B, Smith HM, Springer PS. LATERAL ORGAN BOUNDARIES defines a new family of DNA-binding transcription factors and can interact with specific bHLH proteins. Nucleic Acids Res. 2007;35:666371. https://doi.org/10.1093/nar/gkm775.

57. Makkena S, Lamb RS. The bHLH transcription factor SPATULA regulates root growth by controlling the size of the root meristem. BMC Plant Biol. 2013; 13:1. https://doi.org/10.1186/1471-2229-13-1.

58. Ohashi IK, Saegusa M, Iwamoto K, Oda Y, Katayama H, Kojima M, et al. A bHLH complex activates vascular cell division via cytokinin action in root apical meristem. Curr Biol. 2014;24:2053-8. https://doi.org/10.1016/j.cub. 2014.07.050

59. Katayama H, Iwamoto K, Kariya Y, Asakawa T, Kan T, Fukuda H, et al. A negative feedback loop controlling bHLH complexes is involved in vascular cell division and differentiation in the root apical meristem. Curr Biol. 2015; 25:3144-50. https://doi.org/10.1016/j.cub.2015.10.051.

60. Mace ES, Singh V, Van Oosterom EJ, Hammer GL, Hunt CH, Jordan DR. QTL for nodal root angle in sorghum (Sorghum bicolor L. Moench) co-locate 
with QTL for traits associated with drought adaptation. Theor Appl Genet. 2012;124:97-109. https://doi.org/10.1007/s00122-011-1690-9.

61. Uga Y, Okuno K, Yano M. Drol a major QTL involved in deep rooting of rice under upland field conditions. J Exp Bot. 2011;62:2485-94. https://doi.org/ 10.1093/jxb/erq429.

62. Uga Y, Hanzawa E, Nagai S, Sasaki K, Yano M, Sato T. Identification of aSOR1 a major rice QTL involved in soil-surface rooting in paddy fields. Theor Appl Genet. 2012;124:75-86. https://doi.org/10.1007/s00122-011-1688-3.

63. Uga Y, Yamamoto E, Kanno N, Kawai S, Mizubayashi T, Fukuoka S. A major QTL controlling deep rooting on rice chromosome 4. Sci Rep. 2013;3:3040. https://doi.org/10.1038/srep03040.

64. Uga Y, Kitomi Y, Yamamoto E, Kanno N, Kawai S, Mizubayashi T, et al. A QTL for root growth angle on rice chromosome 7 is involved in the genetic pathway of DEEPER ROOTING 1. Rice. 2015;8:8. https://doi.org/10.1186/ S12284-015-0044-7.

65. Hamada A, Nitta M, Nasuda S, Kato K, Fujita M, Matsunaka H, et al. Novel QTLs for growth angle of seminal roots in wheat (Triticum aestivum L.). Plant Soil. 2012;354:395-405. https://doi.org/10.1007/s11104-011-1075-5.

66. Christopher J, Christopher M, Jennings R, Jones S, Fletcher S, Borrell A, et al. QTL for root angle and number in a population developed from bread wheats (Triticum aestivum) with contrasting adaptation to water-limited environments. Theor Appl Genet. 2013;126:1563-74. https://doi.org/10.1007/ s00122-013-2074-0.

67. Collard BC, Mackill DJ. Marker-assisted selection: An approach for precision plant breeding in the twenty-first century. Philos Trans R Soc Lond Ser B Biol Sci. 2008;363:557-72. https://doi.org/10.1098/rstb.2007.2170.

68. Steele KA, Price AH, Shashidhar HE, Witcombe JR. Marker-assisted selection to introgress rice QTLs controlling root traits into an Indian upland rice variety. Theor Appl Genet. 2006;112:208-21. https://doi.org/10.1007/s00122005-0110-4.

69. Steele KA, Price AH, Witcombe JR, Shrestha R, Singh BN, Gibbons JM, et al. QTLS associated with root traits increase yield in upland rice when transferred through marker-assisted selection. Theor Appl Genet. 2013;126: 101-8. https://doi.org/10.1007/s00122-012-1963-y.

70. Meuwissen TH, Hayes BJ, Goddard ME. Prediction of total genetic value using genome-wide dense marker maps. Genetics. 2001;157:1819-29 https://www.ncbi.nlm.nih.gov/pmc/articles/PMC1461589/.

71. Pace J, Gardner C, Romay C, Ganapathysubramanian B, Lubberstedt T. Genomewide association analysis of seedling root development in maize (Zea mays $\mathrm{L}$ ). BMC Genomics. 2015;16:47. https:/doi.org/10.1186/s12864-015-1226-9.

72. Biscarini F, Cozzi P, Casella L, Riccardi P, Vattari A, Orasen G, et al. Genomewide association study for traits related to plant and grain morphology and root architecture in temperate rice accessions. PLoS One. 2016;11:e0155425. https://doi.org/10.1371/journal.pone.0155425.

73. Aysha K, Abdul W, Snowdon R, Friedt W. Genetic dissection of root architectural traits by QTL and genome-wide association mapping in rapeseed (Brassica napus). Plant Breed. 2019;138:184-92. https://doi.org/10. 1111/pbr.12665.

74. Arifuzzaman M, Oladzadabbasabadi A, McClean P, Rahman M. Shovelomics for phenotyping root architectural traits of rapeseed/canola (Brassica napus L.) and genome-wide association mapping. Mol Gen Genomics. 2019;294: 985-1000. https://doi.org/10.1007/s00438-019-01563-x.

75. Ayalew H, Liu H, Borner A, Kobiljski B, Liu C, Yan G. Genome-wide association mapping of major root length QTLs under PEG induced water stress in wheat. Front Plant Sci. 2018;9:1759. https://doi.org/10.3389/fpls. 2018.01759.

76. Liu P, Jin YR, Liu JD, Liu CY, Yao HP, Luo FY, et al. Genome-wide association mapping of root system architecture traits in common wheat (Triticum aestivum L.). Euphytica. 2019;215:121-32. https://doi.org/10.1007/s10681-0192452-z.

77. An H, Dong H, Wu T, Wang Y, Xu X, Zhang $X$, et al. Root growth angle: An important trait that influences the deep rooting of apple rootstocks. Sci Hortic. 2017;216:256-63. https://doi.org/10.1016/j.scienta.2017.01.019.

78. Abu-Abied M, Szwerdszarf D, Mordehaev I, Yaniv Y, Levinkron S, Rubinstein $M$, et al. Gene expression profiling in juvenile and mature cuttings of eucalyptus grandis reveals the importance of microtubule remodeling during adventitious root formation. BMC Genomics. 2014;15:826. https://doi. org/10.1186/1471-2164-15-826

79. Vilasboa J, da Costa CT, Fett-Neto AG. Rooting of eucalypt cuttings as a problem-solving oriented model in plant biology. Prog Biophys Mol Biol. 2019;146:85-97. https://doi.org/10.1016/j.pbiomolbio.2018.12.007.
80. Xu L. De novo root regeneration from leaf explants: wounding, auxin, and cell fate transition. Curr Opin Plant Biol. 2018;41:39-45. https://doi.org/10. 1016/j.pbi.2017.08.004

81. Xu X, Li X, Hu X, Wu T, Wang Y, Xu X, et al. High miR156 expression is required for auxin-induced adventitious root formation via MxSPL26 independent of PINs and ARFs in Malus xiaojinensis. Front Plant Sci. 2017;8: 1059. https://doi.org/10.3389/fpls.2017.01059.

82. Roychoudhry S, Del Bianco M, Kieffer M, Kepinski S. Auxin controls gravitropic setpoint angle in higher plant lateral branches. Curr Biol. 2013; 23(15):1497-504. https://doi.org/10.1016/j.cub.2013.06.034.

83. Kitomi Y, Hanzawa E, Kuya N, Inoue H, Hara N, Kawai S, et al. Root angle modifications by the DRO1 homolog improve rice yields in saline paddy fields. Proc Natl Acad Sci U S A. 2020;117(35):21242-50. https://doi.org/10. 1073/pnas.2005911117.

84. Kosoy A, Pagans S, Espinas ML, Azorin F, Bernues J. GAGA factor downregulates its own promoter. J Biol Chem. 2002;277(44):42280-8. https://doi. org/10.1074/jbc.M20750520082.

85. Bai B, Wang L, Lee M, Zhang Y, Rahmadsyah AY, Ye BQ, et al. Genome-wide identification of markers for selecting higher oil content in oil palm. BMC Plant Biol. 2017;17:93. https://doi.org/10.1186/s12870-017-1045-z.

86. Nishio S, Terakami S, Matsumoto T, Yamamoto T, Takada N, Kato H, et al. Identification of QTLs for agronomic traits in the Japanese chestnut (Castanea crenata Sieb. Et Zucc.) breeding. Hortic J. 2018;87:43-54. https:// doi.org/10.2503/hortj.OKD-093.

87. Werner CR, Voss-Fels KP, Miller CN, Qian W, Hua W, Guan C-Y, et al. Effective genomic selection in a narrow-gene pool crop with low-density markers: Asian rapeseed as an example. Plant Genome. 2018;11(2). https://doi.org/10. 3835/plantgenome2017.09.0084.

88. Mason RE, Addison CK, Babar A, Acuna A, Lozada D, Subramanian N, et al. Diagnostic markers for vernalization and photoperiod loci improve genomic selection for grain yield and spectral reflectance in wheat. Crop Sci. 2018;58: 242-52. https://doi.org/10.2135/cropsci2017.06.0348.

89. Hansey CN, Vaillancourt B, Sekhon RS, de Leon N, Kaeppler SM, Buell CR. Maize (Zea mays L.) genome diversity as revealed by RNA-sequencing. PLoS One. 2012;7(3):e33071. https://doi.org/10.1371/journal.pone.0033071.

90. Zheng W, Shen F, Wang, W, Wu B, Wang X, Xiao C et al. Quantitative trait loci-based genomics-assisted prediction for the degree of apple fruitcover color. Plant Genome 2020; e20047. https://doi.org/10.1002/tpg2.20047.

91. Pilcher RLR, Celton JM, Gardiner SE, Tustin DS. Genetic markers linked to the dwarfing trait of apple rootstock 'Malling 9'. J Am Soc Hortic Sci. 2008;133: 100-6. https://doi.org/10.21273/JASHS.133.1.100.

92. Fazio G, Wan YZ, Kviklys D, Romero L, Adams R, Strickland D, et al. Dw2, a new dwarfing locus in apple rootstocks and its relationship to induction of early bearing in apple scions. J Am Soc Hortic Sci. 2014;139(2):87-98. https://doi.org/10.21273/JASHS.139.2.87.

93. Harrison N, Harrison RJ, Barber-Perez N, Cascant-Lopez E, Cobo-Medina M, Lipska $\mathrm{M}$, et al. A new three-locus model for rootstock-induced dwarfing in apple revealed by genetic mapping of root bark percentage. J Exp Bot. 2016;67(6):1871-181. https://doi.org/10.1093/jxb/erw001.

94. Foster TM, Celton JM, Chagne D, Tustin DS, Gardiner SE. Two quantitative trait loci, Dw1 and Dw2, are primarily responsible for rootstock-induced dwarfing in apple. Hortic Res. 2015;2:15001. https://doi.org/10.1038/hortres.2015.1.

95. Daccord N, Celton JM, Linsmith G, Becker C, Choisne N, Schijlen E, et al. High-quality de novo assembly of the apple genome and methylome dynamics of early fruit development. Nat Genet. 2017;49:1099-106. https:// doi.org/10.1038/ng.3886.

96. Shen F, Huang Z, Zhang B, Wang Y, Zhang X, Wu T, et al. Mapping gene markers for apple fruit ring rot disease resistance using a multi-omics approach. G3. 2019;9:1663-78. https://doi.org/10.1534/g3.119.400167.

97. Li H, Handsaker B, Wysoker A, Fennell T, Ruan J, Homer N, et al. The sequence alignment/map format and SAMtools. Bioinformatics. 2009;25: 2078-9. https://doi.org/10.1093/bioinformatics/btp352.

98. Magwene PM, Willis JH, Kelly JK. The statistics of bulk segregant analysis using next generation sequencing. PLoS Comput Biol. 2011;7:e1002255. https://doi.org/10.1371/journal.pcbi.1002255.

99. Moser C, Gatto P, Moser M, Pindo M, Velasco R. Isolation of functional RNA from small amounts of different grape and apple tissues. Mol Biotechnol. 2004;26:95-100. https://doi.org/10.1385/MB:26:2:95.

100. Kim D, Langmead B, Salzberg SL. HISAT: a fast spliced aligner with low memory requirements. Nat Methods. 2015;12:357-60. https://doi.org/10. 1038/nmeth.3317. 
101. Pertea M, Pertea GM, Antonescu CM, Chang TC, Mendell JT, Salzberg SL. StringTie enables improved reconstruction of a transcriptome from RNA-seq reads. Nat Biotechnol. 2015;33:290-5. https://doi.org/10.1038/nbt.3122.

102. Love MI, Huber W, Anders S. Moderated estimation of fold change and dispersion for RNA-seq data with DESeq2. Genome Biol. 2014;15:550. https://doi.org/10.1186/s13059-014-0550-8.

103. Conesa A, Gotz S, Garcia-Gomez JM, Terol J, Talon M, Robles M. Blast2GO: a universal tool for annotation visualization and analysis in functional genomics research. Bioinformatics. 2005;21:3674-6. https//doi.org/10.1093/bioinformatics/bti610.

104. Xie C, Mao X, Huang J, Ding Y, Wu J, Dong S, et al. KOBAS 2.0: A web server for annotation and identification of enriched pathways and diseases. Nucleic Acids Res. 2011;39:316-22. https://doi.org/10.1093/nar/gkr483.

105. Da L, Liu Y, Yang J, Tian T, She J, Ma X, et al. AppleMDO: a multi-dimensional omics database for apple co-expression networks and chromatin states. Front Plant Sci. 2019;10:1333. https://doi.org/10.3389/fpls.2019.01333.

106. Liu J, Shen F, Xiao Y, Fang HC, Qiu CP, Li W, et al. Genomics-assisted prediction of salt and alkali tolerances and functional marker development in apple rootstocks. BMC Genomics. 2020;21 (1):550. https://doi.org/10.1186/ s12864-020-06961-9.

107. Zhang M, LV Y, Wang Y, Rose JK, Shen F, Han Z, et al. TATA box insertion provides a selection mechanism underpinning adaptations to Fe deficiency. Plant Physiol. 2017;173:715-27. https://doi.org/10.1104/pp.16.01504.

108. Hellens RP, Allan AC, Friel EN, Bolitho K, Grafton K, Templeton MD, et al. Transient expression vectors for functional genomics, quantification of promoter activity and RNA silencing in plants. Plant Methods. 2005;1:13. https://doi.org/10.1186/1746-4811-1-13.

109. Tian J, Han ZY, Zhang J, Hu Y, Song T, Yao Y. The balance of expression of dihydroflavonol 4-reductase and flavonol synthase regulates flavonoid biosynthesis and red foliage coloration in crabapples. Sci Rep. 2015;5:12228. https://doi.org/10.1038/srep12228.

110. Hu DG, Li YY, Zhang QY, Li M, Sun CH, Yu JQ, et al. R2R3-MYB transcription factor MdMYB73 is involved in malate accumulation and vacuolar acidification in apple. Plant J. 2017;91:443-54. https://doi.org/10.1111/tpj.13579.

111. Schütze K, Harter K, Chaban C. Bimolecular fluorescence complementation (BiFC) to study protein-protein interactions in living plant cells. Methods Mol Biol. 2009;479:189-202. https://doi.org/10.1007/978-1-59745-289-2_12.

\section{Publisher's Note}

Springer Nature remains neutral with regard to jurisdictional claims in published maps and institutional affiliations.

Ready to submit your research? Choose BMC and benefit from:

- fast, convenient online submission

- thorough peer review by experienced researchers in your field

- rapid publication on acceptance

- support for research data, including large and complex data types

- gold Open Access which fosters wider collaboration and increased citations

- maximum visibility for your research: over $100 \mathrm{M}$ website views per year

At $\mathrm{BMC}$, research is always in progress.

Learn more biomedcentral.com/submissions 